\title{
Meeting Report \\ Organ-on-Chip in Development: Towards a Roadmap for Organs-on-Chip
}

doi:10.14573/altex.1908271

\section{Introduction}

Organ-on-Chip is considered a potentially game-changing technology born from the convergence of tissue engineering and microfluidic technology. Organ-on-Chip devices (OoCs) are expected to offer effective solutions to persisting problems in drug development and personalized disease treatments. This report surveys the current landscape in research, development, application, and market opportunities for OoCs towards establishing a global and multi-stakeholder OoC ecosystem. Based on panel discussions held at the Vision Workshop (Stuttgart, 23 May 2018) organized by the EU ORCHID consortium, as well as on additional bibliometric study, market analysis, and expert interviews conducted within the EU ORCHID project, we outline perceived unmet needs, key challenges, barriers, and perspectives of the field. We finally propose recommendations towards the definition of a comprehensive roadmap that could render OoCs realistic models of human (patho)physiology in the near future.

\subsection{The healthcare challenge and the OoC roadmap}

A critical problem in the development of effective disease treatments is the paucity of adequate model systems to identify drug targets, screen toxicity, predict clinical drug efficacy, and ascertain the effects of active substances in humans (Marx et al., 2016). Traditional animal models (McGonigle and Ruggeri, 2014; Smirnova et al., 2018) or conventional cell cultures (Pampalonie et al., 2007; Watson et al., 2017) often do not accurately mimic human physiology and thus tend to poorly recapitulate human disease pathophysiology or accurately predict in vivo responses to medical treatments. This is a major cause of late drug failures in clinical trials, high costs of new drugs, and lack of medication for some diseases (Marx et al., 2016). In addition, translational issues (McGonigle and Ruggeri, 2014; Smirnova et al., 2018) and ethical questions raised by animal use increase the pressure to minimize animal experimentation. For these reasons, the pharmaceutical industry is looking for new ways to improve the drug development process (Esch et al., 2015), assess drug toxicity (Ewart et al., 2018), and identify effective and personalized treatments; biomedical researchers require better systems to model diseases to improve understanding of their mechanism and etiology; and cosmetics, chemical, food and other industries are in need of physiologically relevant human models to determine toxicological and other hazard risks of various substances under increasingly stringent regulatory requirements (Marx et al., 2016).

Organ-on-Chip (OoC) is increasingly regarded as a potentially game-changing technology for these problems (Bahinski et al., 2015) and able to meet the needs of different stakeholders (Middelkamp et al., 2016). In spite of its promise (Zhang and Radisic, 2017), pharma has nevertheless remained cautious to invest in this new technology, presently awaiting evidence of its added cost-benefit value and whether it could represent a feasible route to precision medicine and improved patient stratification. It is thus necessary to bridge the gap between the potential of OoC systems and their worldwide acceptance. Defining the putative benefits of OoCs and how these can be proven and achieved is the preamble for an OoC roadmap - which is one of the aims of the ORCHID project.

The Horizon 2020 FET-Open project Organ-on-Chip In Development (ORCHID) started in 2017 with the goal of creating a roadmap for OoC technology and of building a network of academic, research, industrial, and regulatory institutions to move OoCs from laboratories into general use to benefit the citizens of Europe and beyond. The ORCHID Consortium is a collaboration between 7 partner organizations from 6 European countries - namely: Leiden University Medical Center (coordinator, The Netherlands), Institute for Human Organ and Disease Model Technologies (hDMT, The Netherlands), Delft University of Technology (TU Delft, The Netherlands), Commissariat à l'Energie Atomique et aux Energies Alternatives (CEA, France), imec (Belgium), Fraunhofer Institute for Interfacial Engineering and Biotechnology (Fraunhofer IGB, Germany), and University of Zaragoza (Spain) - and engages an international advisory board of world-renowned experts from the OoC field ${ }^{1}$. As part of the project, these and other experts were asked to share their views on the state-of-the-art, unmet needs, challenges, and barriers of the field. The results of the expert interviews (refer to Section A.2 of Appendix ${ }^{2}$ for the full list of interviewees and experts that contributed to ORCHID), together with the results of bibliographical, bibliometric, and market analyses of OoC technology (see the Appendix ${ }^{2}$ for a description of the analytical methodology), were combined with the insights and conclusions of the ORCHID Vision Workshop, held in Stuttgart on May 23, 2018. The goal of the workshop was to define the pillars of a European OoC roadmap, including the

\footnotetext{
1 https://www.h2020-orchid.eu

2 doi:10.14573/altex.1908271s
} 


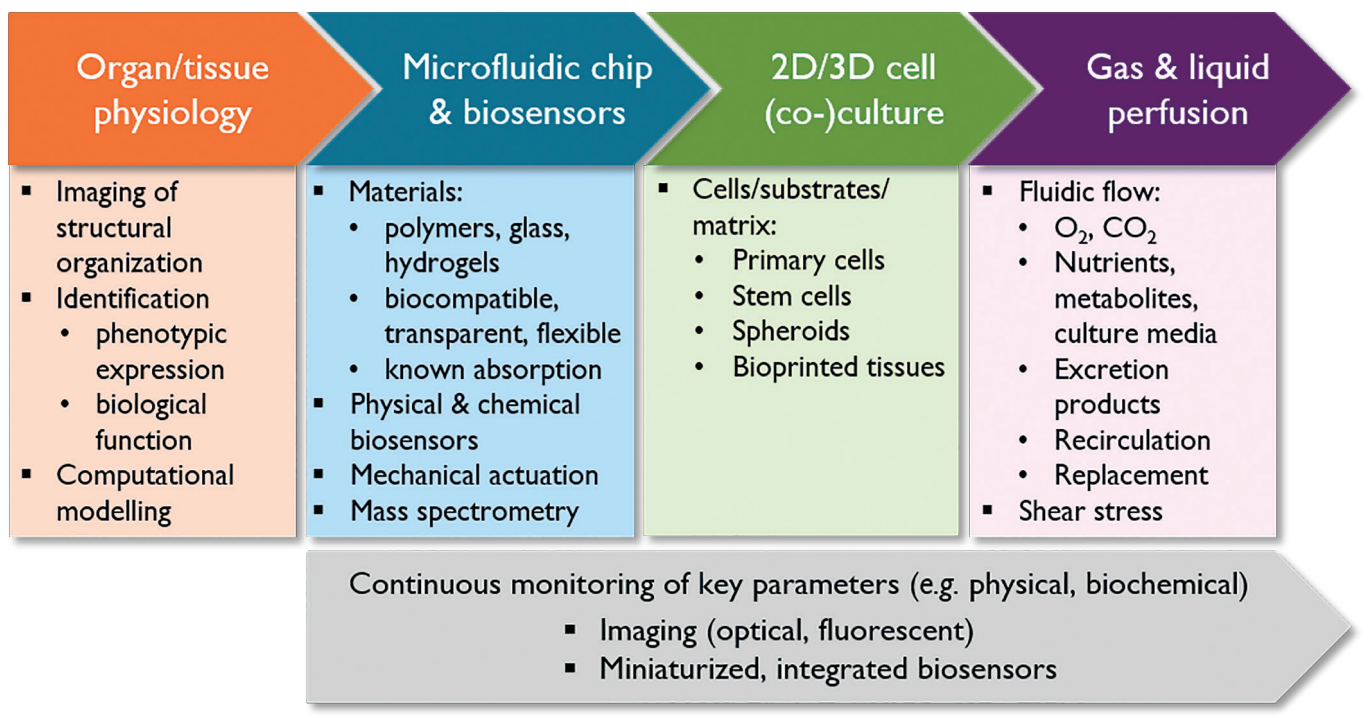

Fig. 1: Simplified synopsis of the OoC value chain, according to the ORCHID analyses

definition of specific goals and their feasibility for concrete deployment of the OoC technology.

This paper outlines the results of the Vision workshop and of ORCHID's updated analyses, discussions among experts, conclusions and recommendations, with a view to creating a roadmap fostering a thriving global OoC ecosystem. An early draft of this document ${ }^{3}$ was released at the International OoC Symposium (IOOCS, Eindhoven, the Netherlands, 8-9 November 2018). The present version features extended content and reference list, and discloses the full list of contributing experts (see Appendix ${ }^{2}$ ).

\subsection{Organ-on-Chip: Definition, key features, and value chain}

According to the interviewed experts, and as ratified during the ORCHID workshop, an OoC can be defined as "a fit-for-purpose microfluidic device, containing living engineered organ substructures in a controlled microenvironment, that recapitulates one or more aspects of the organ's dynamics, functionality and (patho) physiological response in vivo under real-time monitoring".

OoCs can be classified into two types with complementary goals and distinct complexity: (i) single-organ systems, emulating key functions of single tissues or organs, and (ii) multi-organ platforms, combining multiple OoCs to reproduce the systemic interaction and response of several organ models within a single system. Multi-OoCs link individual OoCs through tubing or microfluidic channels (Vernetti et al., 2017), often coated with endothelial cells or with functional coupling provided by transfer of effluent from one organ's effluent reservoir to another's input res- ervoir. In either case, the coupling mimics in vivo physiological coupling and provides appropriate cell-to-fluid volume ratios and flow distributions to create realistic in vitro models of subsystems of the human body (Ronaldson-Bouchard and Vunjak-Novakovic, 2018). Human(-body)-on-Chip (HoC) systems go beyond this and aim to emulate whole organismal physiology by integrating many relevant single-organ models (Marx et al., 2016).

As implied by these definitions, OoC technology is poised on converging advances in tissue engineering, semiconductor and polymer microfabrication, and human cell sourcing. The associated value chain emerging from the ORCHID analyses (Fig. 1) highlights the need for multidisciplinary approaches to implement OoCs and for facilitating dialogue between academic and industrial developers as well as stakeholders such as clinicians, patients, regulators and different end-users. All these disciplines are represented in the ORCHID Consortium.

\section{State-of-the-art}

\subsection{An internationally expanding field of research}

The OoC field emerged from the convergence of microfluidics and tissue engineering research. Whereas in the early 2000's, patents and publications in the field mainly focused on microfluidics and associated fabrication techniques, more recently advances in stem cell biology, combined with decades of fundamental biological studies in cell signaling and biomechanics, have accelerated the development of OoCs.

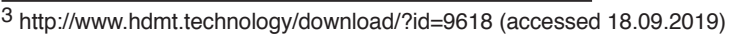


The ORCHID bibliometric approach reflected this increasing activity by finding evidence for a rise in both dedicated patents and publications with a compound annual growth rate (CAGR) of $+46 \%$ over the last ten years (123 patents and publications in 2007, compared to at least 390 in 2017) (Fig. 2a). Such continuous growth, driven by the huge market potential, was made possible by the concomitant diffusion of multidisciplinary approaches supported by improved dialogue between developers such as biologists (cell culture, physiology), engineers (microfluidics, biosensing systems), material scientists (microenvironment, substrates), but also regulators, patients, clinicians, and end users, from both academia and industry. Originating in the USA, which pioneered the development of customized cellular microenvironments to capture the structural complexity of organs, this worldwide interest is increasingly engaging Europe, which has a strong track record in tissue engineering and microfluidics, and for which OoCs could represent a new growth opportunity (Fig. 2b,c). The Asia-Pacific region (APAC) also contributes substantially to the field with both established contributors and newcomers, supported by exponential growth in technological and biological research, especially in Korea, China and Japan.

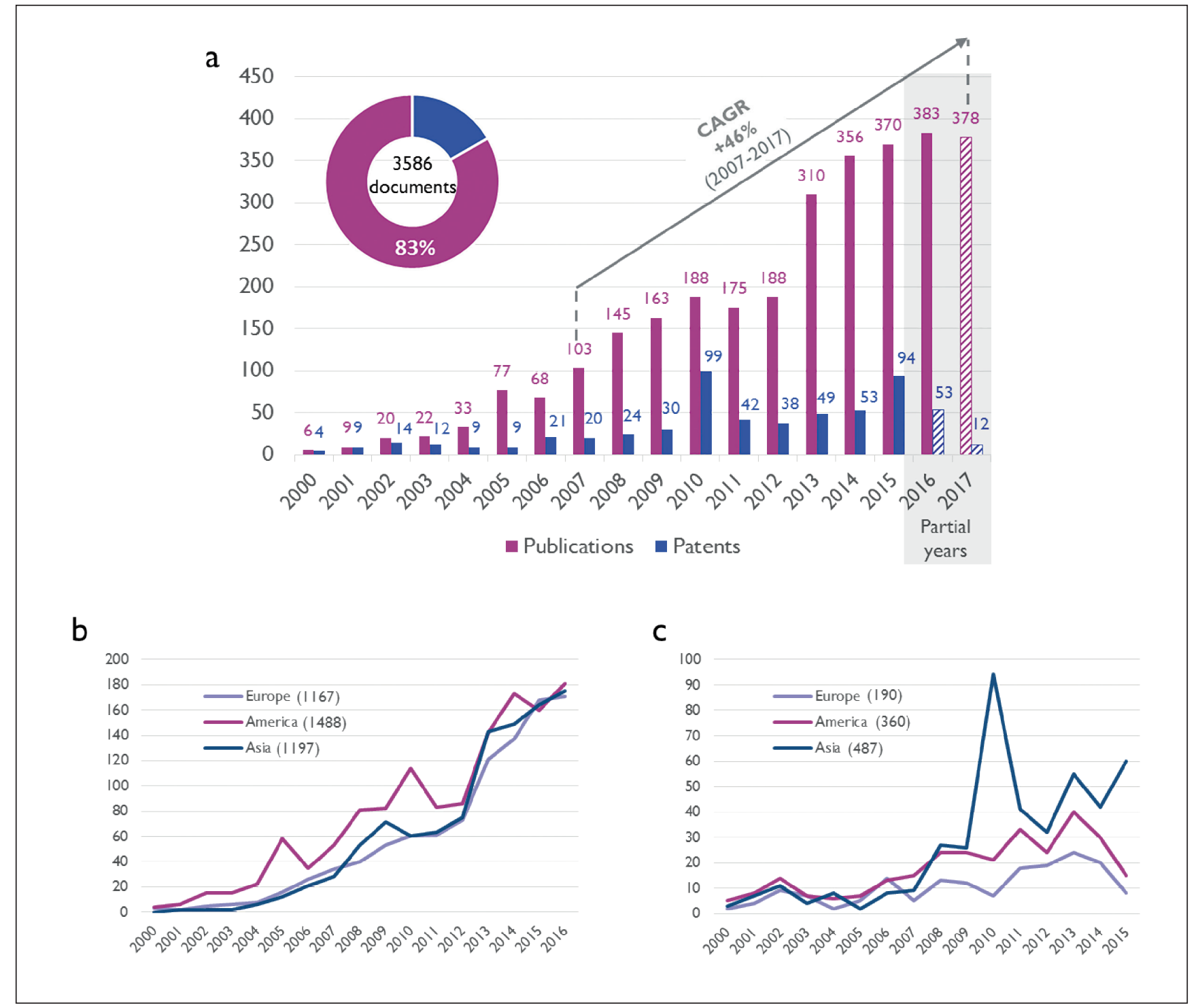

Fig. 2: The ORCHID bibliographic analysis

a) Temporal evolution of the number of OoC-related patents and scientific publications over the 2000-2017 period. The imported database includes 3586 documents, mostly publications ( $83 \%$ versus $17 \%$ of patents). The 592 identified patents include granted (38.9\%), pending $(25.8 \%)$ and fallen or revoked (35.3\%) patents. Year 2015: latest complete year for patents (18 months are needed to publish the patent application). Year 2016: latest complete year for publications (documents were imported in November 2017). CAGR, Compound Annual Growth Rate. b) Temporal evolution of the number of publications per region. c) Temporal evolution of the number of patents per region. 


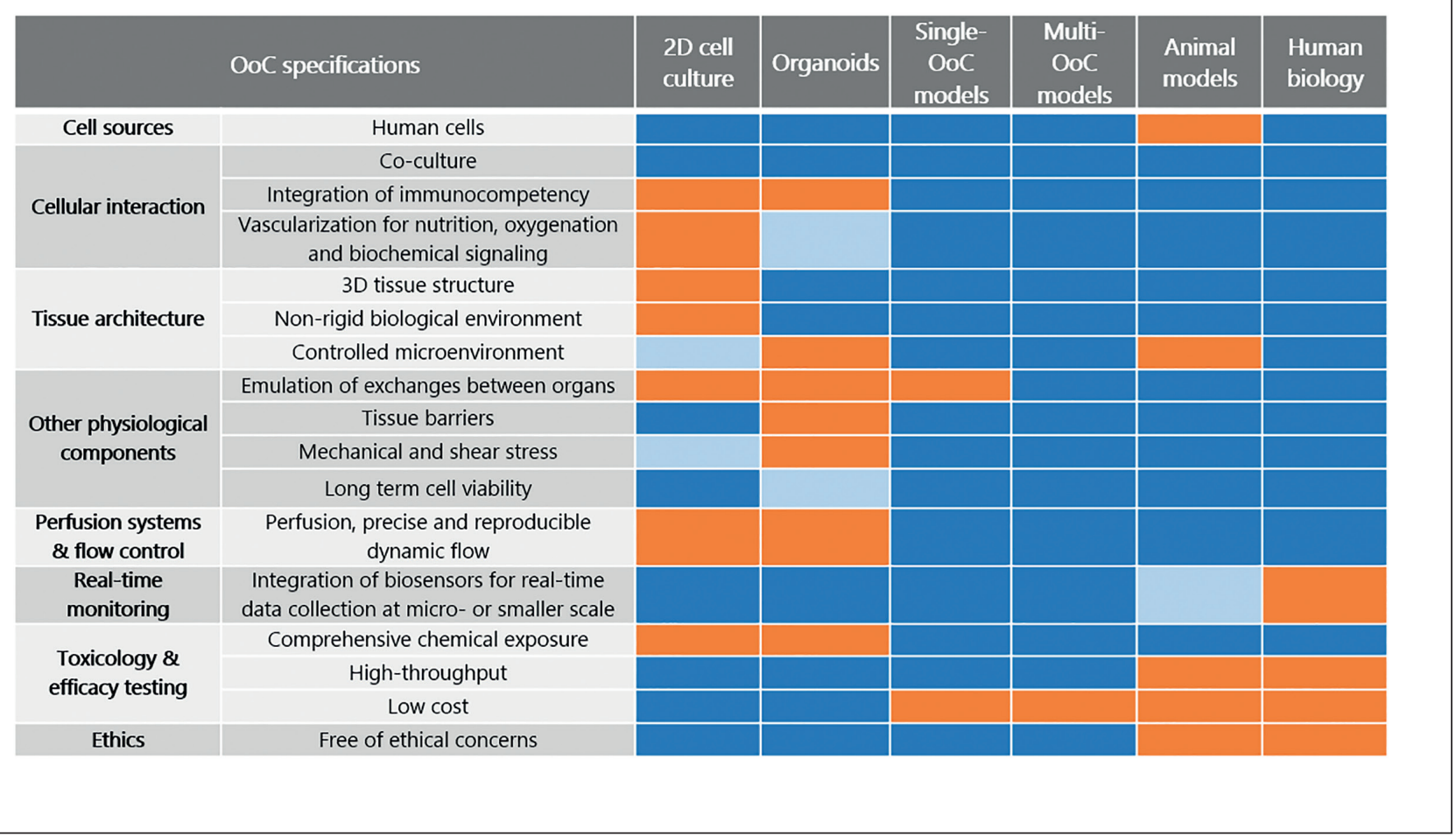

\section{Fig. 3: Comparison of key specifications and features across available biological models}

Animal models can include human cells - consider for instance patient-derived xenografts (PDXs) in nude mice, and nude mice with humanized immune systems. Biosensors include TEER, genetic reporter cells, cell impedance spectroscopy, and microphysiometry. Orange boxes, not yet demonstrated; light blue boxes, possible or envisioned in the near future; blue boxes, in use.

\subsection{OoCs hold promise as avatars for native functional tissues}

Dynamic R\&D activities have been translated into technological advances in both microfluidics and tissue engineering, providing OoCs with key added values towards more accurate views of what happens in the human body compared to other models (Fig. 3). OoC technology allows reconstitution of the microarchitecture of the organ supported by the design of a dedicated mechanical context matching the shape, surface pattern and stiffness of organ-specific microenvironments. Precise control over microfluidic flow rate enables optimal oxygenation and nutrition supply, and affords not only long-term viability of healthy tissues but also an efficient circulation of immune cells, antibodies, biochemical signaling molecules and metabolites, and the ability to collect small volumes of fluid containing secreted cell products for analyses. Continuous perfusion and mechanical stress help to build dynamic tissue models, which are physiologically more relevant than conventional static cell cultures and enable spatiotemporal control of chemical gradients and mechanical cues to study the influence of the microenvironment on the cells. Moreover, OoCs allow precise investigation of specific tissue-tissue interfaces, organ-organ communication and biological events that cannot be monitored in animals or human patients. Their small size, ability to control the microenvironment, optical access at high spatial resolution, inte- gration of biosensors for real-time data collection, and possibility of interfacing with both targeted (Vernetti et al., 2017) and untargeted mass spectrometry (Brown et al., 2016) provide OoCs with important advantages over other models (Marx et al., 2016; Wikswo et al., 2013a). The tightly monitored regulation of the cellular environment and homeostasis should facilitate long-term cell culture, possibly over periods of months. In addition, the great diversity and range of complexity achievable in OoCs offer the opportunity to optimize or even customize the design for targeted studies, paving the way to personalized medicine.

In light of these potential benefits, the main applications of OoCs are seen to range from toxicity testing to human disease model building and drug discovery. Users are increasingly from a wider spectrum of fields and include not only biomedical researchers attempting to understand disease mechanisms and etiology but also industrials with different profiles - pharmaceutics, biotechnology, cosmetics, chemistry and environment - all interested in predicting efficacy and safety of compounds for humans. Some researchers are even further, already exploiting aspects of the long-term potential of OoCs for studying developmental biology principles and optimizing differentiation of iPSCs into various terminally differentiated cell types in situ for application to and understanding of regenerative medicine and tissue replacement purposes. Existing models and proposed applications have 
been comprehensively reviewed previously (Marx et al., 2016; Zhang and Radisic, 2017; Ronaldson-Bouchard and Vunjak-Novakovic, 2018; Zhang et al., 2018).

Nevertheless, OoCs remain in need of substantial scientific evidence of correlation of readouts with clinical and human physiological behavior. In particular, the metrics to be considered and their corresponding readouts largely remain to be defined. They are a prerequisite for demonstrating the relevance of OoCs compared to other traditional approaches (e.g., single cell type, single monolayer; see also Section 3.1) and to develop a roadmap of how they could be implemented in decision-making processes. Encouraging instances in the recent literature include verification of detectable penetration of trimethylamine-N-oxide in human cerebrospinal fluid by Del Rio et al. (2017), first predicted by Vernetti et al. (2017) using a multi-OoC system, and the joint work of the Wyss Institute and Janssen Pharmaceuticals on modelling the toxicity of a monoclonal antibody therapeutic (Barrile et al., 2018) (see Section 4.1).

\subsection{Rapid development of OoCs driven by academics supplying multifaceted industrials}

The OoC market gathers many of its participants from different disciplines, and continuously evolves due to rapid technological advances and the field's strong interdisciplinary requirements. The ORCHID bibliometric analysis identified at least 650 players (the top 30 are shown in Fig. 4). Many of the most active ac- ademic teams are concentrated within pioneering American hubs such as the ecosystems of Boston (Wyss Institute, MIT, Harvard, Brigham and Women's Hospital) and California (Berkeley, UCLA, UCI, Stanford), and the universities of New York (Cornell, Columbia) and Pennsylvania (Drexel, Pittsburgh). These hubs have tended to work especially on brain, lung and heart to address toxicological issues, but also on muscle, vasculature and bone marrow. The APAC region has historically included players like the University of Seoul (Korea), the Chinese Academy of Science (Beijing, China) and the University of Tokyo (Japan), leading chemical, physical and biological engineering approaches to emulate in particular brain, liver and lung tissues. The attractive market has also driven the repositioning of key European players in microfluidics and cell culture, such as the Fraunhofer Institute (Germany), the University of Twente (The Netherlands), the CEA (France), the Technological University of Compiègne (part of the CNRS, France), Jena University (Germany), and the Swiss Federal Institute of Technology (EPFL, Switzerland), which largely focus their research on the modelling of brain, liver, kidney and skin for toxicological purposes.

These academic ecosystems supply industrials from various domains, which may coexist to play a dedicated role in the OoC value chain (Fig. 5, 6): i) pharmaceutical companies; ii) companies specialized in microfabrication, imaging and electronics/robotics; iii) lab-on-chip manufacturing companies; and iv) OoC companies (i.e., commercializing fully operational OoC). Indeed, the devel-

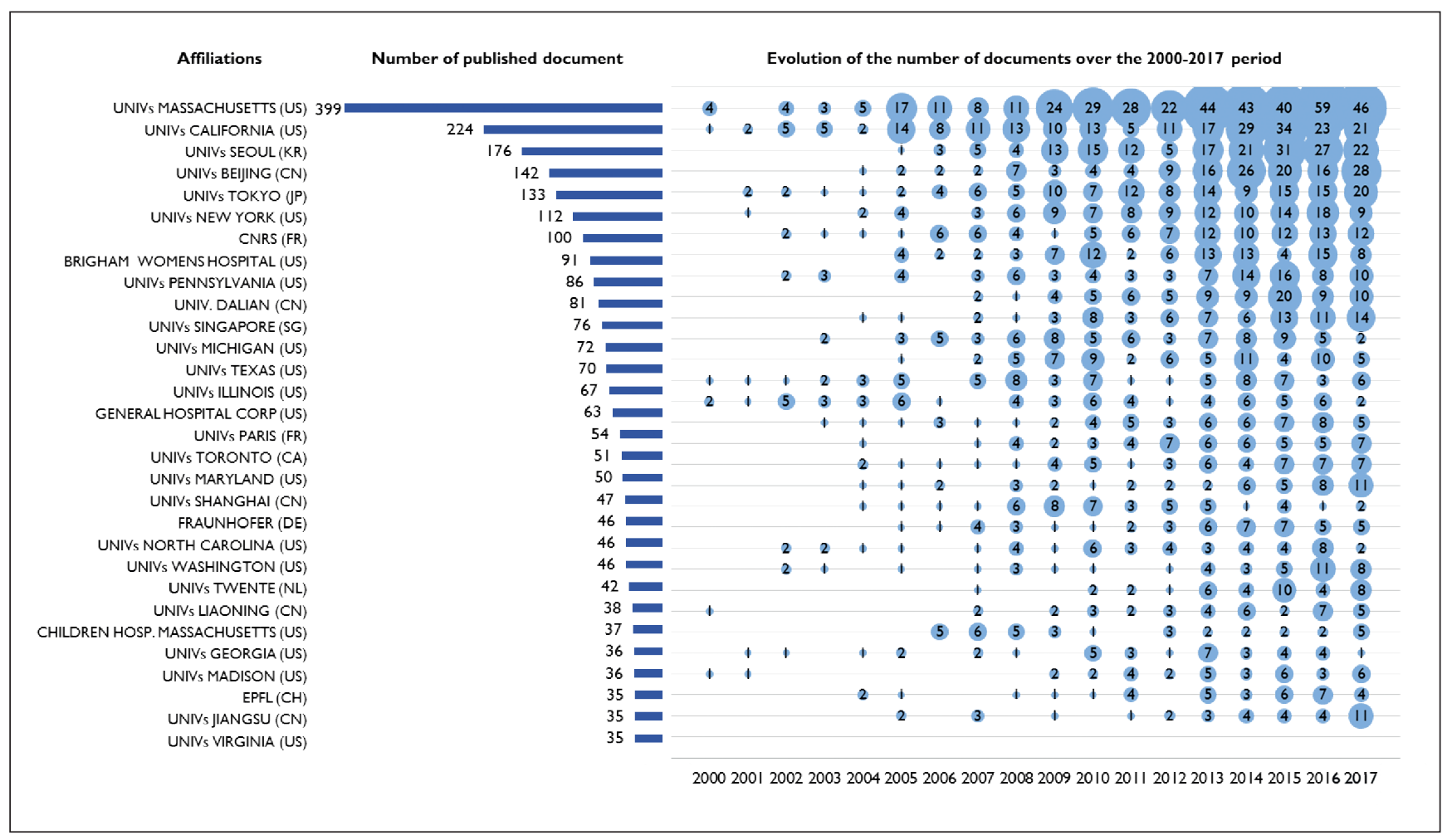

Fig. 4: The top 30 academic players in the OOC field according to the ORCHID bibliographic analysis

These, out of 650 identified academic players, published more than 34 documents over the 2000-2017 period. Blue bars, number of patents and publications. The size of the circles is directly proportional to the number of identified documents published each year. 


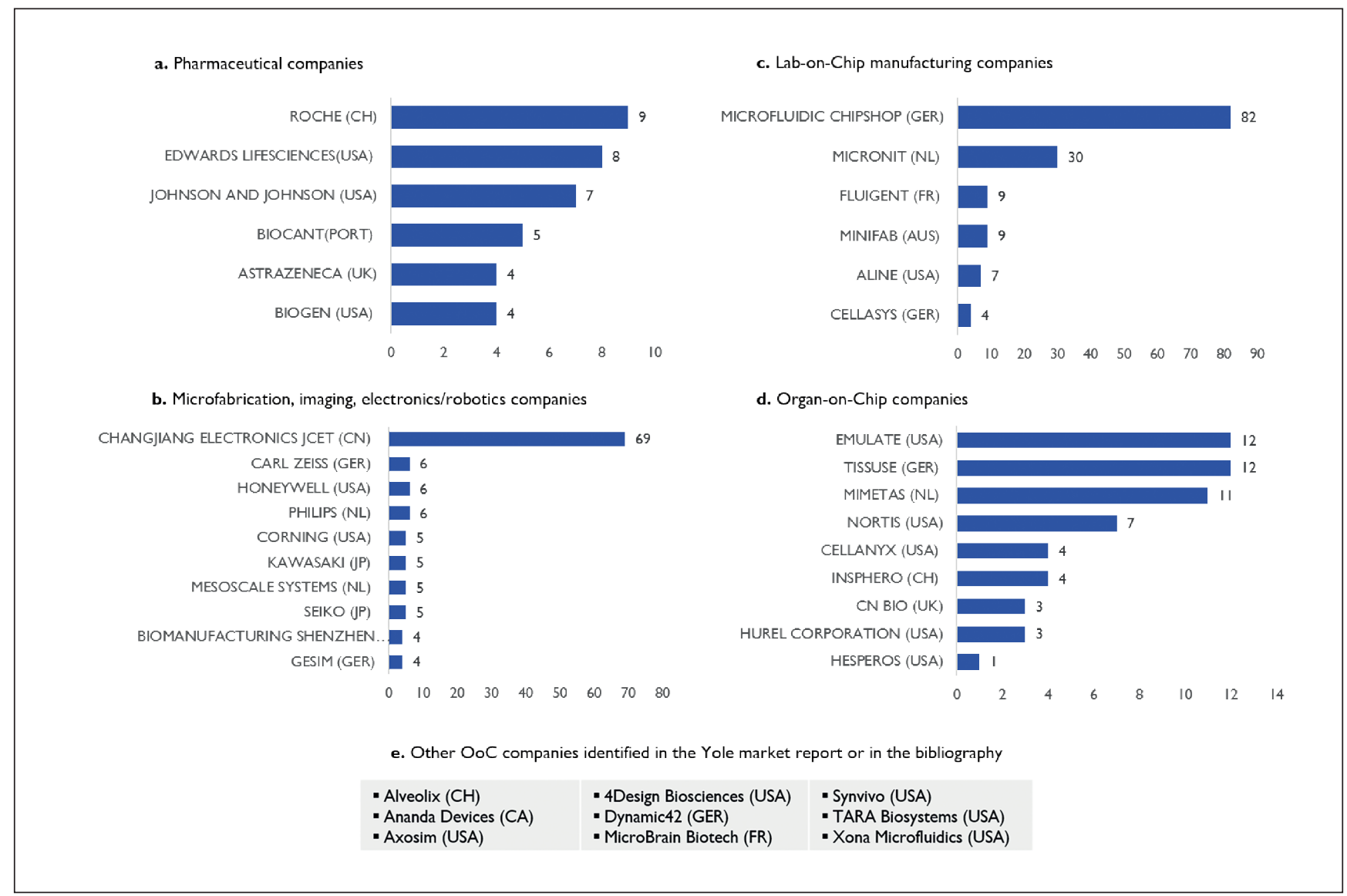

Fig. 5: Overview of the top 30 companies active in the OoC field

Companies are characterized as pharmaceutical (a), lab-on-chip (b), microfabrication, imaging and electronics/robotics (c), OoC companies proper (d) and others (e) - which published more than 2 documents over the 2000-2017 period, according to the ORCHID bibliometric study. Note: Charles Draper Lab is a non-profit and non-stockholding organization with a strong R\&D activity. For this reason, it is not listed among companies.

opment of OoCs has been supported over the past several years by pharmaceutical companies (e.g., GSK, Roche, AstraZeneca) searching for alternative or better predictive models, especially for the lung, the liver, and the digestive and nervous systems. OoC manufacturing and peripheral instrumentation require the concomitant involvement of supply companies specialized in microfabrication, imaging, electronics and robotics (e.g., Seiko, Philips, Carl Zeiss; Fig. 6), in particular for OoC characterization and monitoring. Traditional lab-on-chip manufacturing companies (e.g., Aline, Micronit, Microfluidic Chipshop, Minifab) consider OoCs as a promising growth opportunity. They are starting to partner with OoC companies and propose tailored approaches to support hardware development to scale up and standardize chip production.

OoC companies are mostly start-ups founded by ex-academic teams (e.g., CN Bio, TissUse, Emulate, Mimetas; Fig. 6) (Zhang and Radisic, 2017). According to the Yole Développement market report (Yole (market) report in the following), only 18 private OoC companies were in the market in 2017 (Roussel et al., 2018). Among the most active, TissUse and Nortis are characterized by a multi-tissue R\&D activity, whereas the others have largely prior- itized single-tissue development. Very few of them are already in the production and commercialization phase, the others being predominantly engaged in an iterative process with end users to test different OoC solutions, i.e., manufacturing prototypes and producing small series in-house. For instance, Emulate formed various strategic partnerships with pharmaceutical industries (Roche, Takeda Pharmaceuticals, Merck, Janssen) to improve its solutions, but also with the Food and Drug Administration (FDA) to evaluate and qualify the use of its technology for toxicology testing. OoC companies need both external partners and high financial investments to go through the prototyping phase and to scale up the production. Impressive growth is also made possible by a switch towards the small- or medium-scale production volume, as shown by Mimetas, which is mass-producing its OrganoPlate ${ }^{\circledR}$ after only 4 years of existence, and is supplying nearly all major pharmaceutical companies.

The current market positioning of OoC companies is tightly associated with the type of devices developed and their "technology readiness level". Three different business models are presently distinguishable: i) ready-for-culture microfluidic devices (chips 


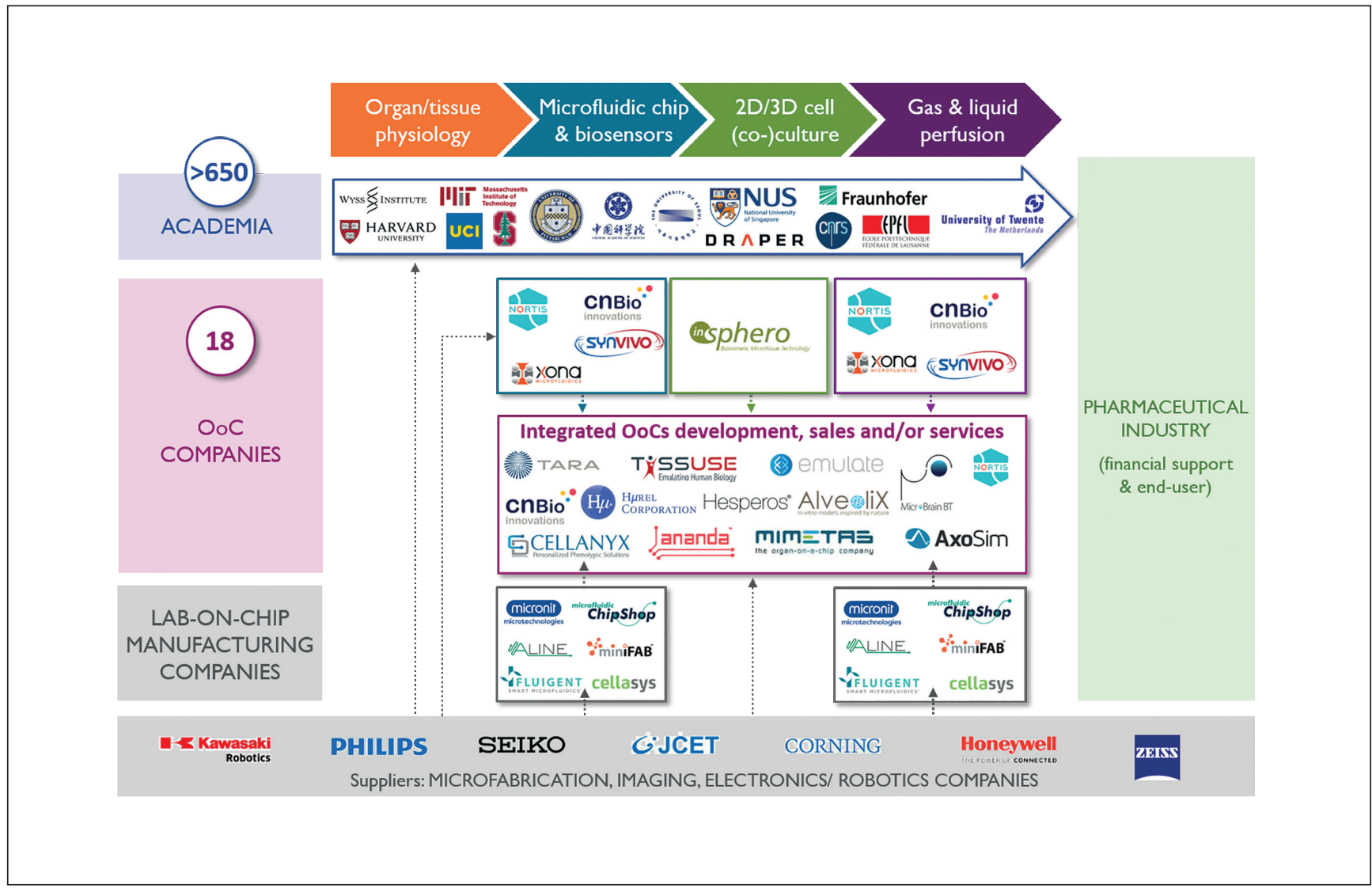

Fig. 6: OoC players' positioning along the value chain (see Fig. 1)

The Figures represent the number of academic players (blue circle) and OoC companies (purple circle) identified via the bibliometric approach. Academics and OoC companies show a dedicated expertise for the development of fully operational OoCs, whereas the other industrials are supporting their development through technological expertise, financing and/or partnerships. For each profile, only the top players are shown. (Note: Charles Draper Lab is a non-profit and non-stockholding organization with a strong R\&D and training/educational activity. Therefore, here it is not considered as a company).

and/or plates); ii) fully operational OoCs, including the microfluidic device and cell culture integration; and/or iii) a full-service offering to perform in-house tests requested by end users. Companies may switch between these business models; and according to the Yole market report, the large majority of OoC companies are not decided whether they should sell the devices or offer testing services (Roussel et al., 2018). Starting with a service-based business model may help to build strong relationships with customers, whereas getting direct input and better understanding of customers' needs enables the joint development of effective OoC platforms. From an end-user point of view, the service-based offer could be of great value for early stage screening, when there is a large number of different compounds to be tested, especially in terms of logistic organization. It is mostly more convenient to ship a drug or a chemical compound than a cell culture. To understand a complex biological interaction, though, some customers, particularly pharmaceutical companies, may be more interested in buying the $\mathrm{OoC}$ to do their experiments in-house.

Whatever the business model of choice, the OoC companies know that minimizing the operational complexity is crucial for commercialization and market penetration. They need to entertain tight relationships with academia to ensure a continuous technological sourcing and to remain competitive.

\subsection{Public and private investments are required to move OoCs from bench to bedside}

Both public and private sources have substantially funded OoC start-ups and research groups during the last few years. For instance, in 2012 the US National Center for Advancing Translational Sciences (NCATS), in conjunction with the National Institutes of Health (NIH) Common Fund, invested \$70M over a 5-year period to launch the Microphysiological Systems or Organs-on-Chips Program (Low and Tagle, 2017). The aim of this program - part of a coordinated effort between the NIH, the Defense Advanced Research Projects Agency (DARPA) and the FDA - was to accelerate the development of human OoCs that "will improve the reliability to identify human drug toxicities and predict the potential efficacy of a drug in a human population prior to use of the drug in late-stage clinical studies" (NCATS website). The program awarded funds to three main multi-partner 


\section{a. Optimistic scenario}

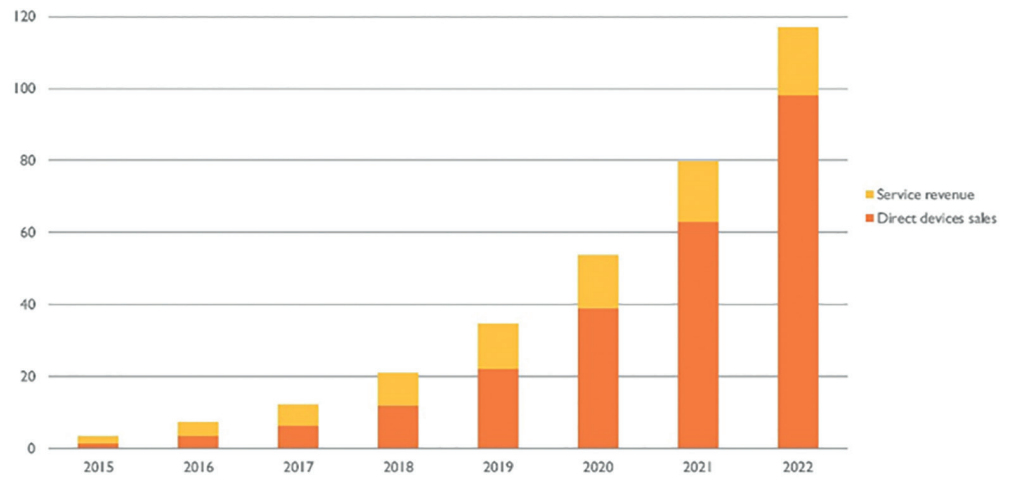

\section{b. Realistic scenario}

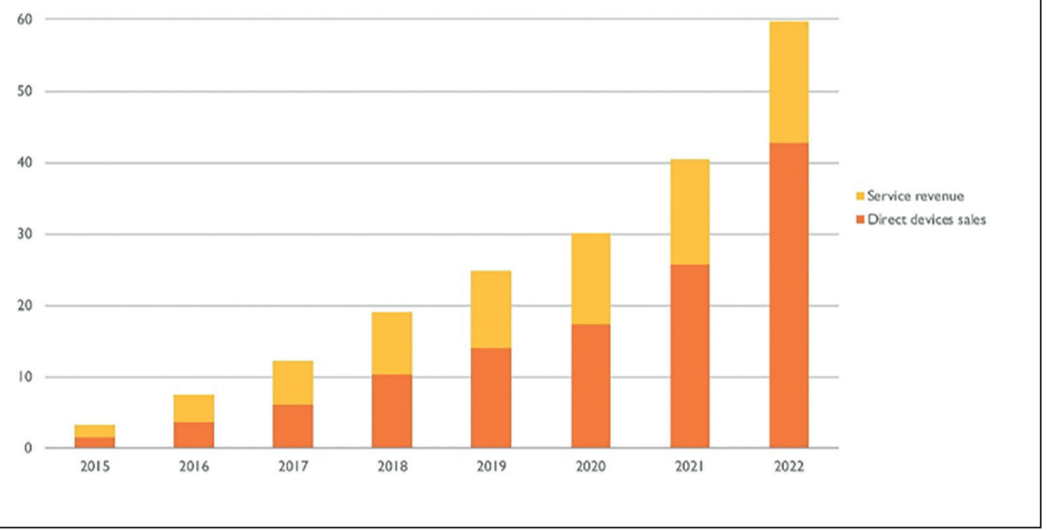

Fig. 7: Market forecasts for OoC devices over the 2017-2022 period proposed by the Yole Développement market report

The optimistic (a) and realistic (b) OoC scenarios show that the market could grow at an expected CAGR of $57 \%$ to $38 \%$ to reach respectively $\$ 117 \mathrm{M}$ to $\$ 60 \mathrm{M}$ in 2022. The estimated revenues are split between direct devices sales (ready-for-culture devices and fully operational OoCs, orange bars) and fullservice sales (tests performed in-house, yellow bars). CAGR, Compound Annual Growth Rate. (Reproduced with permission from Roussel et al., 2018). initiatives, led respectively by the Wyss Institute and MIT. Similarly, the Space and Naval Warfare Systems Center, Pacific (SSC Pacific), on behalf of the Defense Threat Reduction Agency (DTRA), awarded a \$24M federally funded grant in 2013 to a project led by Wake Forest Baptist Medical Center's Institute for Regenerative Medicine to develop a body-on-a-chip model that could determine responses to harmful agents and aid the development of potential therapies (DTRA eX Vivo Capabilities for Evaluation and Licensure "X.C.E.L." program). In 2014 the DTRA additionally funded the $\$ 19 \mathrm{M}$ multi-institutional project Advanced Tissue-engineered Human Ectypal Network Analyzer (ATHENA) to develop a system interconnecting four organ constructs, namely for liver, heart, lung and kidney.

In the meantime, there is growing confidence among private investors in OoC technology. In particular, 2018 was prolific for fundraising, with $\$ 36 \mathrm{M}$ and $\$ 20.5 \mathrm{M}$ secured by Emulate and Mimetas, respectively. According to the Yole market report, the most efficient company in fundraising is Emulate with 4 rounds in 4 years for a total of \$93M (Roussel et al., 2018). Other US companies have also raised several millions of dollars: these include $\mathrm{H} \mu \mathrm{rel}$ Corporation ( $\$ 9.2 \mathrm{M}$ in 2013), and Nortis (more than $\$ 8 \mathrm{M}$ in total, with a first round of $\$ 2.65 \mathrm{M}$ in 2014). In Europe, 2 companies have also succeeded in raising funds: Mimetas (The Netherlands) with a total of $\$ 27.65 \mathrm{M}$ ( 3 rounds in 2014, 2015 and 2018), and TissUse (Germany) with $\$ 4.6 \mathrm{M}$ in 2015 . The Netherlands funded a national OoC initiative (called NOCI) in 2016 with a budget of $€ 18.8 \mathrm{M}$ for 10 years; a H2020 Innovative Training Network project on OoC (EUROoC), coordinated by Fraunhofer IGB, was recently granted $€ 3.94 \mathrm{M}$ for 4 years. Globally, US companies are far ahead in fundraising: the US government is particularly active in supporting the OoC field, especially through DARPA, NIH, DTRA and the Environmental and Protection Agency (EPA), and most recently the National Aeronautics and Space Administration (NASA) and the Center for the Advancement of Science in Space (CASIS), with investments of more than $\$ 224 \mathrm{M}$ over the past 5 years.

\subsection{Market forecasts: From an emerging market towards exponential growth}

The Yole market report estimates the combined sales of OoC devices and services at no more than $\$ 7.5 \mathrm{M}$ in 2016 , with the potential to undergo an impressive growth and become a multi-billion dollar market in the mid- to long-term, in view of the possibility that OoCs might help the industry to save billions of dollars every year, especially by bridging the translational gap between preclinical and clinical studies required during drug development (Roussel et al., 2018). As already indicated, only few players are cur- 
rently in the production and commercialization phase; and while pharmaceutical and cosmetics companies are engaged in an iterative process to test different $\mathrm{OoC}$ solutions, they are conservative and might need time to adopt the technology widely. This overall context led Yole Développement to detail both a realistic and optimistic scenario, in which the market could grow at a CAGR from $2017-2022$ of $38 \%$ to $57 \%$ to reach $\$ 60 \mathrm{M}$ to $\$ 117 \mathrm{M}$, respectively in 2022 (Fig. 7; for further market data, see Roussel et al., 2018).

In the optimistic scenario, based on the companies' forecasted revenues (Fig. 7a), the Yole report considers that if all conditions are met, i.e., industrial adoption speeds up, OoC companies can overcome technical challenges and upscale production, $858 \mathrm{k}$ units per year could be manufactured by 2022, corresponding to an overall market of $\$ 117 \mathrm{M}$ (Roussel et al., 2018). The service-based market is presently expected to remain much smaller than the device sales market, and whether it will be sustainable in the long-term by OoC start-ups is unclear. However, the service-based market could grow significantly through involvement of contract research organizations (CROs), which provide a multiplicity of services for pharmaceutical companies and other customers, if they begin to take over from OoC developers and offer large-scale or high-throughput services with OoC models.

In the realistic scenario (Fig. 7b), the Yole report forecasts that the majority of OoC developers will face issues if the demand grows (Roussel et al., 2018). It is noted in particular that scaling up the production will likely slow down the growth of OoC companies during the period 2018-2021. Most of them will have to switch from PDMS prototyping and small-series production to large-scale manufacture in other materials (glass, polymer injection molding), requiring redesign steps that will be expensive for the relatively small companies involved. In this scenario, the OoC market will grow from $\$ 7.5 \mathrm{M}$ in 2016 to $\$ 59.7 \mathrm{M}$ in 2022 because only few companies have already managed to scale up their production. The revenue fraction deriving from services is higher than for the optimistic scenario because OoC companies will focus on customized services to offset losses due to the costs of production upscaling.

\section{Challenges}

\subsection{Unmet needs}

\section{Evidence for added value}

A notable unmet need that emerged from the experts' interviews is data-supported evidence of actual advantages of the OoC technology compared to existing conventional models or well-established approaches to tissue engineering. Particularly, while the superiority of 3D over 2D cell cultures for, e.g., phenotypic expression, is attested in recent scientific literature (Alépée et al., 2014), the advantages deriving from the inclusion of dynamic perfusion and in situ stimulation in OoCs are only recently becoming evident (Vernetti et al., 2017; Del Rio et al., 2017) and arguably need more substantial support. In this context, incorporation of organoids (or dissociated tissue cells derived from them) may have particular advantages, since they represent a renewable source of cell types that are normally difficult to access repeatedly and which have greater similarities to normal or diseased human tissue than the widely used immortalized cell lines.

\section{OoC can pave the way to personalized medicine and de-risking drug development}

The limited ability of preclinical cell cultures and animal models to predict drug efficacy and safety reliably during the later human clinical trial stage (Abaci and Shuler, 2015) is not only disappointing but also ends up wasting billions of dollars each year in development costs for ineffective drugs and slows down the introduction of new medical treatments in the clinic. Spending on drug development increased by orders of magnitude over the past 20 years, but the number of drugs approved for market per year actually declined (Zhang and Radisic, 2017). Many compounds with high potential health benefit are eliminated early in development due to the poor predictability of preclinical models, i.e., they may well be effective in humans but not in animal models as well as the converse, which is more frequent. Some drugs have actually been withdrawn shortly after entering the market because their toxicity (mainly on heart, liver and kidney) was not predicted. Late-stage failures cause catastrophic losses while significantly driving up cumulative costs and patient risk. If OoCs could be positioned as proven predictive tools for preclinical screening, they might represent the ideal solution for the pharmaceutical industry to eliminate ineffective drug candidates as early as possible and curb the costs of drug development (Zhang and Radisic, 2017).

As expected, the OoC applications most cited in scientific literature and in the ORCHID expert interviews lie in the pharmaceutical drug development process (Abaci and Shuler, 2015), and are related largely to safety assessment and efficacy testing (see also Watson et al., 2017). The main idea shared by all players is that OoCs may enable more rapid, accurate, cost-effective, and clinically relevant testing of drugs. According to a recent scenario-based cost analysis conducted in the context of the ORCHID project, experts expect a positive budget impact, reaching a reduction of up to $26 \%$ in the total R\&D costs for drug development (Franzen et al., 2019). While all cost drivers may be impacted, savings would mostly be achieved by improving the success rates for effective disease treatment. The $R \& D$ phases in which experts expect the most benefits are lead optimization and preclinical phases (see also Watson et al., 2017).

Data from animal studies are often poorly indicative of the human situation (McGonigle and Ruggeri, 2014; Mak et al., 2014), since animal preclinical models have limitations in mimicking the complex processes specifically occurring within the human body (Marx et al., 2016). OoC models may potentially bridge the translational gap from target or lead compound discovery to market introduction and actually foster the implementation of the $3 \mathrm{Rs}$, i.e., Reduction, Refinement, and Replacement of animal testing. However, all interviewed experts agreed that OoCs (and most other in vitro systems) are currently far from replacing all animal models, and should be viewed mostly as complementary approaches to animal testing. Specifically, and as evidenced in the ORCHID impact analysis (Franzen et al., 2019), high-throughput plate-based microphysiological systems may find use in target identification, lead selection, and lead optimization at assay scale; chip-based, 
low-to-medium throughput devices may find most fitting use in pre-clinical, single-organ toxicity, or efficacy tests; and multi-organ or coupled OoC systems may target the replacement of animal models for toxicity screening, testing of pro-drugs, and phase I / phase II clinical testing in patients - which is being prefigured as (pre)clinical-trials-on-chip.

Replacing (pre)clinical trials and advancing towards the implementation of personalized medicine are the grand unmet needs targeted by multi-OoC and $\mathrm{HoC}$ systems. OoCs pave the way to personalized medicine approaches by enabling the use of patient-specific primary and stem cell sources to capture important differences arising from genetic diversity, origin, gender or age. For instance, OoCs can enable the development of in vitro clinical trials for patient populations not fit for standard clinical trial designs (i.e., rare and/or pediatric diseases) or to develop drug regimens that are optimized for specific patient biology (Ronaldson-Bouchard and Vunjak-Novakovic, 2018). Along this path, OoCs may represent models not only capable of capturing prior organ(ismal) knowledge and recapitulating known physiological responses, but also, and even more importantly, unique tools for unprecedented investigations and new discoveries for the further advancement of physiology and medical science.

\section{OoCs can benefit toxicology testing}

The interviewed experts share the opinion that industries developing cosmetic, chemical and agro-food consumer products could find huge potential in $\mathrm{OoC}$ applications with respect to toxicological hazard and risk assessment of substances for the study of, e.g., metabolism, effects of toxicants including nanomaterials, collective responses and allergies (see also Zhang and Radisic, 2017). Regulatory requirements for chemicals and cosmetics do not accept any significant hazard potential of substances used for humans. Moreover, there is rising interest in alternative models that allow skin sensitization assessment and systemic toxicity testing for regulatory use, because all animal testing for cosmetic and fragrance products marketed in Europe was banned in 2013. China is meanwhile changing regulations about animal testing for cosmetics, approved the first non-animal tested cosmetics in 2016, and the authorities are being lobbied to encourage reducing animal use. The USA has included a ban on animal testing in their 2007 roadmap for all industries, including the pharmaceutical, chemical and cosmetics industries (Roussel et al., 2018). The chemical industry is also actively working on such strategies, especially on in vitro skin sensitization testing, to avoid unnecessary reassessment of chemicals due to the Registration, Evaluation, Authorization and Restrictions of Chemicals (REACH) regulation (Marx et al., 2016). Nevertheless, more formal validation efforts will be needed prior to full industrial OoC adoption in the chemical and cosmetics industries.

Though not mentioned by any ORCHID interviewee, the tobacco industry could represent another OoC targeted segment in the near future due to the high pressure to increase the safety of its product portfolio (Marx et al., 2016). Ethical considerations have prompted the tobacco industry to develop new approaches and tools to assess smoke-related adverse effects on the human respiratory system, primarily airways and alveoli. OoCs could rep- resent excellent tools to investigate the onset of lung diseases (Benam et al., 2016), such as smoking-induced chronic obstructive pulmonary disease and its malignant transformation.

\subsection{The future of OoCs lies in automation, robustness and integration of complexity \\ Automation and ease of use}

Present OoCs are typically complicated pieces of engineering, whereby the microfluidic chip accommodating the cell co-cultures is connected to external control and supply peripherals. For this reason, ease of use of the systems and increased automation in setting up cell cultures and keeping them viable throughout study times are commonly perceived unmet needs in laboratory practice. Ideal OoCs would be simple devices displaying user-friendly interfaces that facilitate the work of users with even limited training on, e.g., cell cultures and instrumentation. These devices should be easily transferrable from developers to end users by means of standardized guidelines concerning biomarkers and endpoints. In addition, they may be prepopulated with frozen cell cultures ready-to-use by end users upon thaw.

\section{Improving single OoCs}

Among the many different organs and tissue types that can be currently emulated (Marx et al., 2016), the outcome of the experts' interviews and of the ORCHID bibliometric analysis indicated a strong preference for ADME (absorption, distribution, metabolism, excretion) pathway processing, including metabolic (liver, kidney) and digestive organs (Maschmeyer et al., 2015), along with cardiac tissue, lung and central nervous system tissues coupled to a blood-brain barrier substitute (Bhatia and Ingber, 2014). Organ-specific needs to be met included, among others: for the liver, the development of models making use of hepatocytes derived from human induced pluripotent stem cells (hiPSCs; see also next Section for optimal cell sourcing); for the heart, efficient maturation protocols for hiPSC-derived cardiomyocytes to capture the expression of adult phenotypes; for skin, establishing methods to derive all skin cells from hiPSCs, including, e.g., dermal papilla cells needed for hair generation (Abaci et al., 2017). OoC devices can perfuse vessels, thereby including the vitally important flow of a blood surrogate to feed nutrients and remove excreted products through vasculature, and the accompanying shear stress. The experts emphasized that, while immunogenicity testing of drug candidates in animals is essentially obsolete, immune and endocrine systems (Cyr et al., 2017) are missing components required to improve the physiological relevance of OoCs (van de Stolpe and Kauffmann, 2015). Different academic groups are indeed developing bone marrow-on-chip to integrate aspects of immunocompetence in OoC-based drug safety and efficacy testing (Marx et al., 2016). Moreover, predictive models for gametogenesis and testicular toxicity, an infrequent but severe cause of arrest of drug development or "black-box labelling", and for other specific toxicities for which no satisfactory pre-clinical model exists are presumed to be valuable as a niche market for OoCs.

The ORCHID bibliometric analysis highlighted high interest in OoCs as cancer model systems, considered potentially helpful for understanding tumor progression, metastasis formation, and in- 


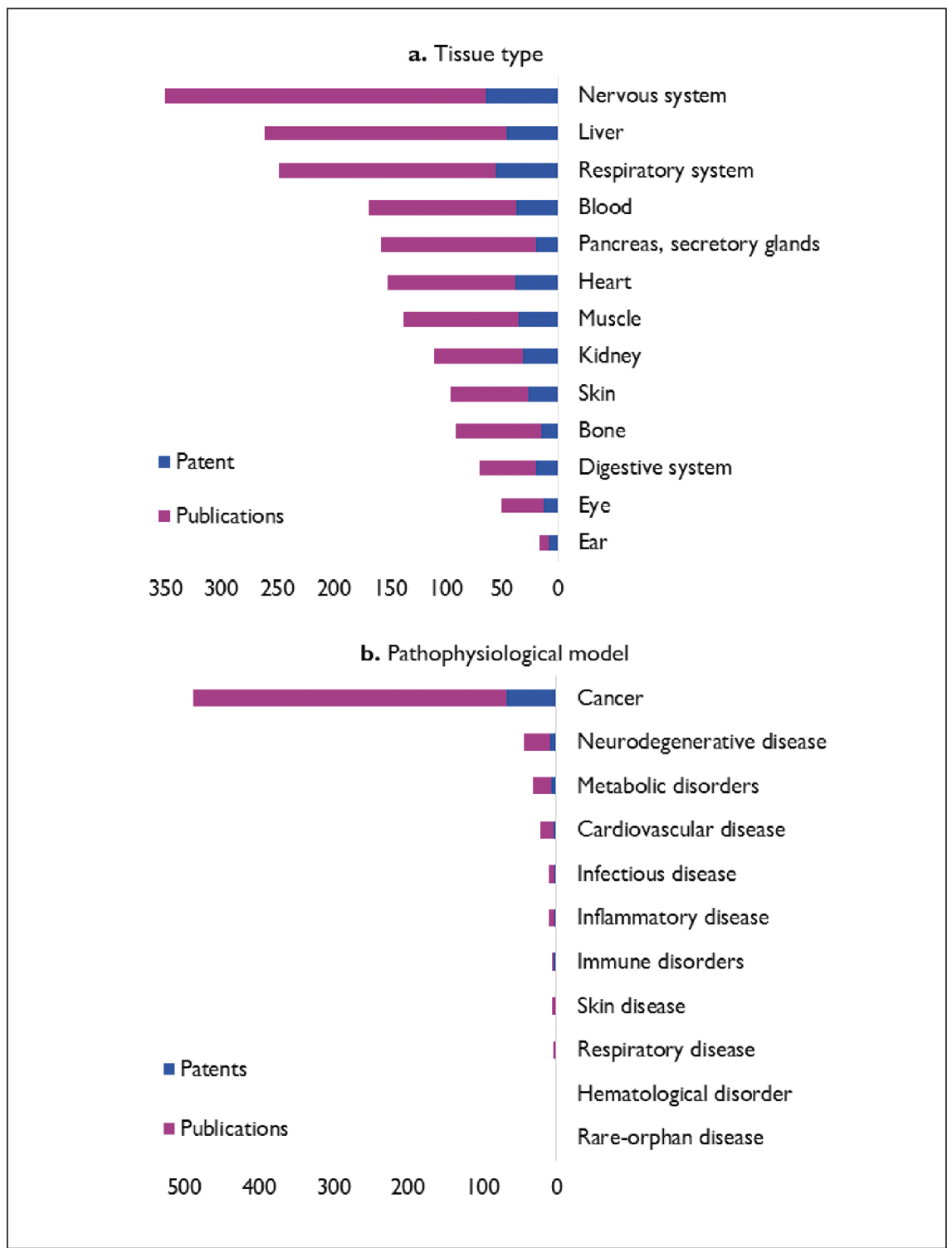

Fig. 8: Global trends in pathophysiological models and tissue types according to the ORCHID bibliometric analysis The imported database of patents and scientific publications was segmented with regards to emulated tissue types (a) and pathophysiological models (b) on a worldwide scale. vestigation of inhibiting strategies. (Fig. 8). With respect to metastasis modelling, detailed recapitulation of the vascular basement membrane remodeling has not yet been reported. The exponential growth of publications dedicated to OoC cancer models may be related to the fact that OoCs are the only systems to model tumor cell intravasation into a surrogate blood stream or immune cell extravasation into the tumor by combining human micro-perfused 3D tumor models with human vasculature (Marx et al., 2016). Such strong interest is also supported by epidemiological data and the high market potential for cancer treatments.

The expert interviews also indicated that, from a patient perspective, stringent unmet medical needs are represented by ways to improve the treatment of diseases - such as cancer, dementia, kidney and rare diseases - for which there is currently no treatment, insufficient treatment (Osaki et al., 2018), or only treatments that are excessively expensive.
Integrating more complexity within multi-OoCs

As mentioned earlier, the development of multi-organ OoCs is envisioned in the perspective of recapitulating the complexity of human physiology at organism level, which is lost in moving away from animal models. A recent eminent example of a multi-OoC is the Evatar system, which couples Fallopian tubes, uterus, cervix and liver made from human tissues with ovaries from mouse tissue to recapitulate 28-day female hormonal cycles (Xiao et al., 2017).

Early claims of a forthcoming full HoC system appear to be presently more realistically modulated by a technical assessment of the formidable difficulties facing its achievement. The latter include: (1) the onset and maintenance of self-contained homeostasis; (2) accounting for the influence of missing organs (Cyr et al., 2017), particularly the hormone background (e.g., gender and reproductive hormones) and immunocompetent cells; and (3) the identification of (alternative solutions to) a single perfusion me- 
dium shared by the multiple cell types (each with different requirements) within the system. Other challenges for multi-OoC and $\mathrm{HoC}$ systems lie in scaling the relative volumes of the individual OoCs (Wikswo, 2014; Wikso et al., 2013b), the interconnection topology, and perfusion rates which should approximate physiological flow configurations (Ronaldson-Bouchard and Vunjak-Novakovic, 2018). Organs involved in ADME are considered the basis of $\mathrm{HoC}$ models, since they are responsible for significant homeostatic effects and are foreseen in comprehensive organ models for compound testing, for instance in skin and lung models to evaluate compound concentration in urine and blood. Modelling of ADME is considered central also because inappropriate drug distribution is regarded as an important cause of unwanted drug side effects - a global leading cause of death. For this purpose, efforts should focus on combining a set of organs that can match the critical functions needed for drug study with a functional endothelium serving as a selective barrier (Brown et al., 2015, 2016) for transport of drugs and bioactive factors (Ronaldson-Bouchard and Vunjak-Novakovic, 2018).

\subsection{Technical challenges}

\section{Structural materials: alternatives to PDMS?}

Polydimethylsiloxane (PDMS) has largely replaced glass and ceramic as the most commonly used material for fabrication of OoCs. The wide adoption of this type of silicone ensues from a convenient combination of advantages, namely biocompatibility and oxygen permeation (crucial for cell viability), optical transparency (permitting real-time optical inspection), tunable viscoelasticity (which supports mechanical actuation), ease of availability, and straightforward processing (no strict need for specific cleanroom facilities) - including deposition by casting and spin-coating, functionalization, metallization, and patterning by soft lithography and molding (Zhang et al., 2018). The almost unanimously remarked disadvantage of PDMS - though common to most polymers, inc luding the plastics of interconnecting tubes - lies in its lipophilicity (Vernetti et al., 2017) and resulting non-selective absorption of hydrophobic drugs, particularly those with low molecular weight (van Meer et al., 2017). Non-selective absorption complicates the localization of target compounds in the devices and, in the absence of models predicting drug absorption rates to PDMS and bioavailability to cells in the devices, can drastically affect the interpretation of analytical results. Moreover, drug absorption directly affects the choice of how to deliver drugs to OoCs. Drug delivery in OoCs should preferably replicate the physiological application in humans, e.g., direct exposure to cells, inhalation, skin absorption, oral delivery, or intravascular delivery by perfusion in vessels. The limitations of PDMS may be tolerated during device prototyping and characterization, but may hinder future large-scale integration unless effectively modelled. Solutions to the non-selective compound absorption in PDMS face a number of challenges: (1) the search for or cost of application-specific formulations of PDMS or alternative polymers, since most are based on polycarbonate, polystyrene, styrene ethylene butylene styrene (SEBS), polyimide, polyurethane or hydrogels for which intellectual property covers use through patents; or (2) the coating of polymer surfaces with lay- ers of endothelial cells, providing a barrier to drug absorption into the substrate only reproducible if the cell monolayer is confluent.

\section{Optimal cell sourcing and shared culture media}

Access to abundant, good quality human cell sources represents an essential technical aspect of OoCs. Multiple cell sources are currently available, and each cell type presents specific advantages and disadvantages. The highest clinical relevance for humans is expected through use of adult primary cells obtained directly from healthy donors or patients, although this is not the only option (Sutherland et al., 2016). However, genetic variance between individuals may arise since it is rare that cells can be obtained repeatedly from the same donor. Adult human cells cannot always be obtained in large quantities from many tissues (e.g., skin biopsies), or may not be readily accessible through biopsies (e.g., from brain or heart). Adult stem cells can be collected and cultured indefinitely from almost all endodermal organs, e.g., the organs of the gastro-intestinal tract and the lungs; they can also be genetically modified and grown in culture in large quantities, but they are not available from all organs or tissues, and they only represent the epithelial component (i.e., not the stroma or vasculature). Pluripotent stem cells (PSCs), derived from reprogramming of adult somatic cells (human induced PSCs, hiPSCs in short) or derived from early embryos (human embryonic stem cells, hESCs), can give rise essentially to all cell types of the human body, and in the case of hiPSCs can be obtained from any individual (Bellin et al., 2012). On the other hand, the differentiated derivatives are generally phenotypically immature, similar to fetal cells. It is thus a remaining challenge to develop culture conditions for the maturation of such cells, and to use them to model diseases developing after birth (Avior et al., 2016). Spheroids and organoids (Fatehullah et al., 2016) derived from human biopsies and hPSCs are also regarded as sources of pre-organized, higher-order tissues suitable for inclusion in OoCs (Zhang et al., 2017).

While the dynamic microenvironment of OoCs, which attempts to mimic human physiology, can support cell differentiation and maturation better than static cell culture conditions, the co-presence of multiple cell types in the same microphysiological device sharing a common culture medium may cause additional problems. According to the ORCHID experts, the issue of the formulation of a single perfusion medium may be solved by a strict biochemical approach, rather than from simple media mixing. Culture media containing, e.g., high glucose concentrations are standard but may not be appropriate as cells mature, and many application-specific media are proprietary so that their composition is not known. Fetal calf serum, widely used until recently to supplement culture medium with growth and other factors, is presently being largely replaced by chemically defined media but, again, formulations may not suit all cell types in co-culture. Finding common media is an active area of research by media supply companies and others. Blood is used as medium as well despite constraints on its usage for safety and other reasons. Finally, whereas the choice of cell types is generally dependent on the specific organs and applications of interest, a related challenge concerns the appropriate representation of the diversity inherent in the human population in terms of gender, ethnic origin and age. 
Long-term viability and multimodal real-time characterization could revolutionize cell culture utility

With typical sizes of around a few millimeters, OoCs afford distinct advantages in terms of non-invasive organ monitoring and tissue investigation compared to direct investigations in human individuals or patients. The small volumes of tissues and culture media confined within the closed, controlled environment of the devices limit the dilution of xenobiotics and metabolites. Small chip volumes additionally help ensure sterile culture conditions, though they may be also responsible for reduced throughput unless part of multi-well assays for example. A related advantage concerns the potential extended viability of cells in OoC devices compared to standard cultures, enabled by continuous microfluidic nutrient feeding and removal of waste products. Recirculation without a liver or kidney however may entail either use of large media volumes or media replacement. Required model viability is considered by the experts interviewed to be sensibly dependent on the application and specific questions at hand: it can range from a few hours to a few days (e.g., 3 to 8 days) for repeated dose/exposure and acute toxicity testing; up to a few weeks for safety and efficacy testing (e.g., 28 days for systemic testing, in accordance with the Organisation for Economic Co-operation and Development (OECD) guidelines for animal tests) (Maschmeyer et al., 2015); and up to several months for chronic toxicity testing or modelling/ monitoring disease course progression in vitro, in analogy with phase II clinical trials. Tissue viability has been hypothesized as being indefinitely extendable in the presence of self-contained homeostasis, particularly when sustained by vascularized interconnections among several organ modules.

Online characterization of organ- or tissue-specific phenotypes, readouts and endpoints in OoCs may include vascular contraction, cell migration, responses of genetic reporters, and metabolic pathways. The methodology is largely dominated by optical and fluorescence microscopy, which are expected to remain prevalent for the foreseeable future. A challenge for assay imaging systems is translation into high-throughput industrial settings. Incubators at body temperature are commonly used to host OoCs and regulate cell culture parameters, such as $\mathrm{pH}$ and $\mathrm{CO}_{2}$ levels. Composition of perfusion media, fluid flow rates, and gas pressure for pneumatic actuation of flexible membranes are mostly controlled by external, partly automated equipment. Connection of fluidic peripherals to chip or plate devices typically involves silicone, Tygon, PEEK or other flexible tubes, which may cause bubble formation and non-selective drug absorption. Proper utilization of devices and peripherals typically requires specific training of users and is expected to be regulated by standardized guidelines. Including such peripherals as integral components of self-contained OoC devices may also raise issues. According to the experts consulted by ORCHID, OoCs should be consumables whereby real-time sensor readout and system control are simultaneous and aligned. The experts recognized that real-time monitoring of bioreactions in OoCs may revolutionize in vivo studies by increasing the throughput of analyses, and acknowledged the existence at present of a gap between hardware development and the absolute quantification of biological response required, partly because the physical, chemical or electrical responses to bioreactions in OoCs are not fully understood. Therefore, the integration of bio-compatible electrical, chemical or physical sensors and actuators is highly sought-after and widely investigated (Zhang et al., 2017). For instance, trans-epithelial electrical resistance (TEER) (Henry et al., 2017) and fluorescein isothiocyanate (FITC)-dextran permeability (Brown et al., 2015, 2016) are being measured as real-time readouts of tissue barrier functions; and micro-impedance tomography is used to quantify the deflection of membranes induced by breathing motion (Mermoud et al., 2018).

Qualification for contexts of use should be preferred to validation Some form of validation of OoC systems is critical for their broader acceptance and uptake in industrial and clinical settings, particularly in relation to supporting decision-making in a regulatory context. In Guidance Document 34, the OECD defines "validation" as "the process by which the reliability and relevance of a particular approach, method, process or assessment is established for a defined purpose". Although this definition is in principle applicable to OoC devices used for a particular purpose, the context of OECD guidance typically relates to the validation of methods intended to form the basis of internationally recognized test guidelines that can be used in any of the 36 OECD member countries, implemented within a Good Laboratory Practice (GLP) quality system (as exemplified in the OECD's Guidance Document on Good In Vitro Method Practices (GIVIMP)), and satisfying the conditions of Mutual Acceptance of Data (MAD) between jurisdictions and regulatory agencies. Therefore, the validation processes foreseen in this context may be more appropriate for highly standardized and widely applicable methods, and may probably not be suitable for emerging $\mathrm{OoC}$ devices in the near future. Moreover, validation per se is not considered by some experts an appropriate nor meaningful concept in this context, because it implies the existence of an accepted standard to be used to confirm or measure validity. In fact, neither animal models nor human clinical effects can be univocally used as golden standard for validity. Besides, a universal approach to validation is probably unrealistic, and a harmonization across contexts and cultures is expected to take too long and might not be possible at all.

Considering that the application of $\mathrm{OoC}$ devices in the short/medium term is most likely to be in the pharmaceutical sector, and will primarily target specific and well-defined contexts of use, focus should be on the qualification of devices, rather than on validation in the broader sense. "Context of use" refers here to a clearly articulated description delineating the manner and purpose of use of a tool, while "qualification" is understood as arriving at a conclusion that the results of an assessment using a model or assay can be relied on to have a specific interpretation and application in product development and regulatory decision-making. Aiming for qualification of OoC devices was in fact the approach proposed in a recent series of workshops devoted to this important topic - held both in the US by the FDA and in Europe by the European Medicines Agency (EMA)'s Safety Working Party - and it was confirmed in the ORCHID workshop. The typical qualification process applied to OoC devices would foresee a comparison between OoC data and corresponding data derived from conventional preclinical drug development models (e.g., animal models, cell sus- 
pensions) or human clinical data if available. If feasible and appropriate, this will inform assessment of whether $\mathrm{OoC}$ responses correspond to expected behavior, and can thus be used as a reliable predictor of human response.

More generally, it is desirable and expected that OoCs reproduce salient physiological features and functional aspects of organs which recapitulate the human condition and responses to exogenous stimuli such as (candidate) drug molecules (Sutherland et al., 2016). A challenge in qualification practice is therefore the establishment of relevant sets of reference or benchmarking compounds with well-known and properly documented pharmacological action(s) and in vivo effects (e.g., for paracetamol, beta blockers). Initiatives exist - for example, led by the NIH in the USA, or the SEURAT-1 program funded by the European Union ${ }^{4}$ - which aim to establish a public database to collect readout data in very specific and platform-independent formats, useful for both pharmaceutical companies and regulatory agencies. The ORCHID experts suggested that such initiatives may inform an open platform shared by partners in pre-competitive settings, tapping crowdsourcing approaches to, e.g., data analysis and device testing, and crowdfunding to share costs. They may be inspired by examples of shared technology planning and development, such as the former International Technology Roadmap for Semiconductors (ITRS) and the International Roadmap for Devices and Systems (IRDS) ${ }^{5}$.

The current scenario is nonetheless fragmented, as most pharmaceutical companies intend qualification as an internal recognition process that does not need to be translatable to other companies, let alone the whole sector. Accordingly, OoCs need only comply with company-specific qualification protocols using company-specific test sets of compounds, established or under development. Though such compounds are typically proprietary and not easily accessible to academic research groups, the IQ Consortium, a not-for-profit organization of pharmaceutical and biotechnology companies, is compiling a list of reference compounds that might be shared for qualification purposes ${ }^{6}$.

\section{Standardization: when is the right time?}

Standardization of OoC devices will likely contribute to ensuring reproducibility and robustness of results both within a single lab and across different labs; uniformity or compatibility of cell or tissue types and sources; and compatibility among chips or modules fabricated by different developers upon interconnection into a multi-organ system. Commercial suppliers of (differentiated stem) cells, such as Cellular Dynamics (CDI) and NCardia, may contribute to standardization through transparent QA and QC protocols prior to use. Moreover, standardization will aid in the practical incorporation of OoC-based studies into analysis workflows and the utilization of results within decision-making contexts. Thus, a move towards standardization is desirable from an industrial as well as from a regulatory point of view. This objective is however not compatible with the current early stage of academic and commercial development of the majority of devices. The exponential growth of the number of publications on OoCs, which is even greater than that accompanying the Human Genome Project, testifies to the continuous introduction, at least in the academic domain, of novel or updated OoC models which contemplate variations in the substrate technology and/or cell sources and types. The experts interviewed mostly shared the opinion that such wide exploration of solutions is welcome and typical of early stages of technology development. To bring most benefit to the field, academic research should preferably not be hindered by the strict imposition of standards or constraints deriving from intellectual property, even though this would counter the perceived interest of pharmaceutical companies and industrial end users. Additionally, it would be premature and counterproductive to converge towards robustly qualified or standardized manufacturing processes prior to gathering substantial evidence and characterization of the capacity of OoC models to host the intended target (cell and tissue) biology and recapitulate in vivo human physiology and response of interest. Standardization of OoC devices and readouts may emerge at some later stage of the research trajectory, as in the case of other technologies, due to the convergence towards solutions generally considered most convenient or optimal, issues related to intellectual property, reorganization of companies, market conditions, and end-user demands. Finally, standardization may alter the landscape of players in the field by constraining the numbers that can afford to comply with both its financial and engineering aspects.

\section{Barriers and perspectives}

\subsection{From industrial hesitance towards acceptance}

OoC developers expect a large market take-off in the next 2-3 years. This optimism is supported by major recent technical breakthroughs (Zhang et al., 2018), which make OoCs a credible option to provide a variety of end users with more predictive testing models. Once OoC models are available, demand is expected to increase and smoothen the road to applications. On the other hand, focusing on less high-profile applications may provide an additional point of market entry for OoCs.

Nevertheless, a more realistic view of the situation shows that there remain substantial barriers to be crossed. Industry is still testing OoCs in comparison with alternatives (e.g., 3D cell cultures, spheroids, tumoroids, organoids) to identify the models most suited for their needs. Evidence-based convenience of the models is required to encourage industrials, especially pharmaceutical, to integrate $\mathrm{OoCs}$ into near-future routine processes. Although OoCs may be more relevant and accurate than animal models or standard cell cultures, they are still expensive and need costly redesign to suit mass production. In addition, compatibility problems may occur due to the current lack of standardization (e.g., different shapes, interfaces, sizes, tissue types and quality, ways of use);

\footnotetext{
4 http://www.seurat-1.eu/ (accessed 18.09.2019)

5 https://irds.ieee.org/ (accessed 18.09.2019)

6 https://iqconsortium.org/ (accessed 18.09.2019)
} 
and it may take years before OoCs are widely accepted by regulatory agencies. Hence, a pragmatic attitude prevails in the industrial context - which, as for any available alternative, evaluates OoCs either on a fit-for-purpose or on a cost-per-data point benefit they may afford. Accordingly, the new technology may be accepted if it provides simpler or cheaper alternatives to established models while reproducing the same results, or if it affords models for which no alternatives currently exist. An example of the latter is provided by joint research of the Wyss Institute with Janssen to model the toxicity of a monoclonal antibody therapeutic which caused death in patients due to pulmonary embolism in Phase I clinical trials: Janssen had not observed any toxicity in preclinical animal models, whereas the Wyss Institute's OoC could replicate the thrombotic behavior (Barrile et al., 2018). Independent, robust and reproducible qualification of purpose-specific OoC models, excluding the risk of false positives (Watson et al., 2017), will accelerate their acceptance.

According to the Yole market report, in the most likely scenario OoCs will be increasingly adopted by industry, due to their significant advantages over existing solutions in terms of predictivity, cost-saving, and being an alternative to animal testing (Roussel et al., 2018). Newcomers, mostly spin-offs or spin-outs from academia, will appear in the OoC developers' market, but in the meantime some companies will fail. It would not be surprising to see biotech companies and instrument developers, who are providing tools to the pharmaceutical industry, acquiring $\mathrm{OoC}$ developers, because of the excellent synergy between these organizations.

\subsection{Early dialogue to ease regulatory hurdles}

Regulatory aspects can represent another significant barrier towards the broader adoption of OoCs. There is a generic and nonOoC specific need to speed up regulatory validation processes. Examples of prior technologies - such as, e.g., cloning, transplantation, CRISPR-CAS - attest to resistance in the adoption and regulation of new medical options. The decisions of regulatory authorities on the efficacy of novel treatments can have significant repercussions in delaying the acceptance and implementation of those treatments, in making the treatment costs liable to reimbursements to patients, and generally on the societal costs of healthcare. In addition, the costs of the certification procedure, involving an extensive series of tests, may exceed the possibilities of academic research groups. This conversely should provide incentive for company and funding agency involvement in the development of OoCs as well as in early dialogue with regulatory authorities to ensure effective and transparent communication and avoid procedural obstacles at later stages of development. Industrial acceptance typically precedes and drives regulation (Marx et al., 2016). Still, there may be a catch in the transfer of the device development process from initial developers to companies. On the one hand, developers may be in immediate need of funding to advance or characterize their models to an extent sufficient to attract interest of the companies. On the other hand, companies may hesitate to provide funding until the developers have demonstrated that their models fit their specific purposes or that they can overcome the companies' concern of being the first to introduce a new model on the market - a model that later might eventually be revealed as ineffective in predicting effects in humans, or not compliant with future regulations.

\subsection{Keeping expectations realistic}

Industry and government agencies already have huge expectations on a few developers of OoC technology, in particular academic centers like the Wyss Institute (and its commercial arm, Emulate Inc.) and MIT as well as companies such as $\mathrm{CN}$ Bio. These developers were repeatedly awarded funding of several tens of millions of dollars, and are the most visible in the field. The Yole market report suggests that the success of these well-funded organizations might be critical for the future of the OoC area (Roussel et al., 2018). Some industry players believe these organizations cannot fail, whereas some think the gap between prototyping and mass commercialization is too large for immediate success. The consequence of any failure of these field pioneers could be devastating for the $\mathrm{OoC}$ field: besides the high visibility of the failure (media coverage, top-rank universities), loss of confidence from both industry and investors would disrupt the prospects of many small companies.

The interviewed experts echoed similar concerns, and expressed almost unanimously the need to remain realistically optimistic about OoC technology. They generally expect OoC to become a key technology in coming years, yet prescribe remaining very cautious in stating the promises and potential of $\mathrm{OoC}$ technology to the media and general public to avoid the pitfalls of hype dynamics. While the experts do not agree whether the OoC technology is currently the object of a hype, the Human Genome Project, stem cells for regenerative medicine, microarray technology, proteomics and gene therapy are examples of previous programs, initiatives or technologies that risk failure to deliver the high expectations raised at their outset.

In further analogy with these precedents, the amount of funding considered necessary to achieve the field's purported goals is expected to be in the order of hundreds of millions to billions of dollars. A large fraction of the costs relates to the translation of proof-of-concept devices from laboratories to market entry, and the tests prescribed for regulatory approval. OoC-related companies are expected to drive such advances, eventually in partnership with governmental agencies, and they find themselves in a critical position in this respect. Attracting investors and considerable funding is critical to gathering the multidisciplinary technical expertise and building the layered infrastructure that can support the development of prototypes into mass fabrication to meet the industrial demands of quality control and reproducibility. To achieve this, the companies need to rely on advertising, which is susceptible to including overstatements. The latter may raise premature or unmatched societal expectations about a technology, which conversely is generally considered to be still at an early development stage.

A common perception of the interviewed experts is that the lay public easily grasps the core concept of OoC technology, and tends to become quickly (over)excited about the potential to decrease or even replace animal testing. However, "organ-on-chip" as a broad technology-defining name may confuse the public by 


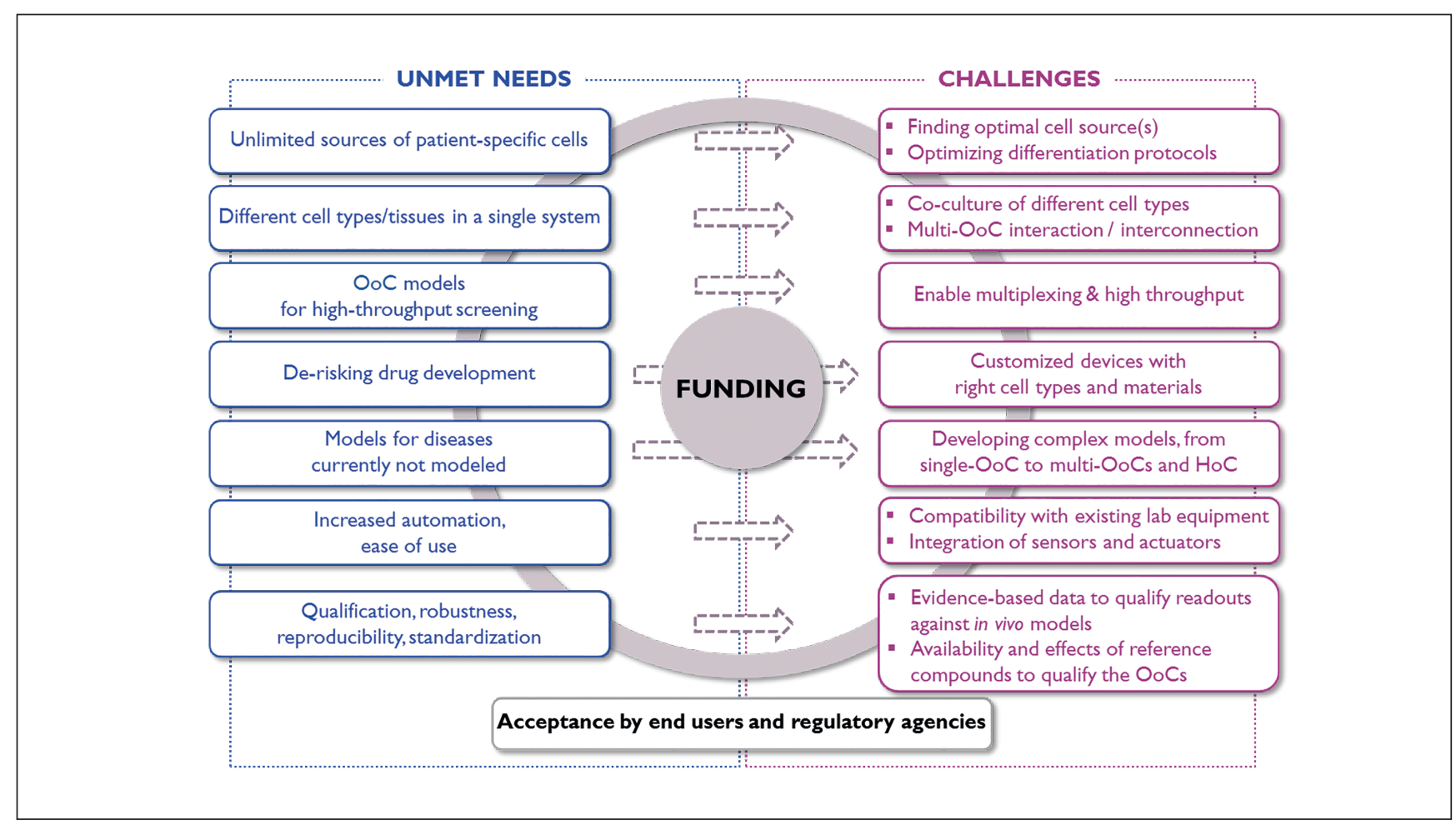

Fig. 9: Key unmet needs and challenges for OoC development

association with other technologies such as artificial organ prostheses, organ replacement or regenerative medicine. The public may not understand how OoC technology actually works and what its limitations are unless browsing specialized journals. On the other hand, the more popular media may tend to cover sensational stories and thus feed the false impression that the technology is already achieving all the advertised promises. An antidote to such perceived distortions may be a synergistic outreach activity by key players and stakeholders based on careful communication of recent achievements and ongoing developments that could inform about actual trends and realistic perspectives.

\subsection{Encouraging early collaboration and education}

The interviewed experts remarked the strong need for integrative programs, collaborative projects, consortia and international funding strategies for the development of OoC technology and applications. Lack of international coordination may be caused by the heterogeneity of the global legislative, regulatory, financial and ethical scenario. Coordination efforts should be supported by a widespread outreach activity, targeting all levels of audience, and by an improved dialogue between regulators, industries, clinicians and patient groups since the early stages of development. In this respect, a digital platform is now being developed within the ORCHID (Mastrangeli et al., 2019) to bring together players (e.g., researchers, laboratories, companies, patient associations, regulators) and to create a community in the OoC field. The platform will evolve over time through the addition of publications, collaborative projects, and additional infor- mation supplied by the members who will progressively become part of it.

Finally, besides topical and field-specific workshops and conferences, incubators of talents are elicited as prime accelerators for the progress of the technology. The experts recommended this should be the pivotal role of institutes, inspired by analogous initiatives of successful information technology companies, where diverse experts could freely interact to traverse linguistic and departmental boundaries and leverage ample resources to develop new ideas and conduct multi-disciplinary research.

\section{Summary and recommendations}

The synergistic convergence of microfabrication technologies and tissue engineering renders OoCs promising tools for the realistic modelling of human physiology and pathology. The aim of an $\mathrm{OoC}$ is not to replicate a whole, living organ but rather to sustain a minimal functional (sub)unit of an organ or tissue that can controllably recapitulate salient aspects of human physiology. For this purpose, as argued in this paper, the main desirable features of an $\mathrm{OoC}$ can be divided into three categories:

(1) Tissue architecture

- Integrated long-term cell culture in defined spatial organizations

- Tissue-tissue interfaces/cell-cell contacts/cellular heterogeneity

- Miniaturization 
(2) Conditions

- Controlled microenvironment (topology, biochemistry, physics)

- Controlled dynamics (fluid flow, electro-mechanical stimuli)

- Continuous automated perfusion

- Real-time monitoring of multiple physical, bio- and electro-chemical parameters

- Automated reproducible multi-sample analysis, comparable with $\mathrm{R} \& \mathrm{D}$ robotics

- Large-scale manufacturability

(3) Functions

- Physio- and pathological relevance

- Recapitulation of organ structure and function

- Recapitulation of dynamic mechano-biological properties and stimuli response of organs

In view of this and of the unmet needs and challenges, ORCHID defined the following recommendations (summarized in Fig. 9) to foster proactivity and progress in this rapidly evolving field (see also Mastrangeli et al., 2019). We remind that the availability of new models and their eventual application or adoption should always be viewed in light of current regulations and rules, such as, e.g., FDA's Animal Efficacy Rule for biodefense applications.

\section{Characterization, qualification and standardization}

1. Do not aim for the whole human: start mimicking single organs first.

2. Demonstrate the benefits and prove with reliable data OoCs' added value compared to other models.

3. Describe the value of OoCs in fit-for-purpose or cost-perdata point scenarios.

4. Develop guidelines for qualification of OoCs through collaboration involving developers, end-users and regulatory bodies.

5. Create lists of reference compounds and related annotations for specific organ effects to qualify OoCs in defined contexts of use, and share the data through a public database.

6. Compare OoC tissue architectures and cellular phenotypes with in vivo tissue histology or histopathology using existing technologies.

7. Only consider standardization of OoCs once there is substantial evidence that these platforms recapitulate in vivo human physiology and responses of interest.

\section{Technology}

8. Integrate real-time, bio-compatible electrical, chemical or physical sensing and analysis, and define and measure the salient parameters that can be predictive.

9. Address the issue of non-selective compound absorption in OoC substrate materials.

10. Minimize operational complexity: develop automation for speeding up $\mathrm{OoC}$ assays, and improve ease of use to enhance reproducibility, robustness, and ease of transfer from developer to end user.
11. Integrate $\mathrm{OoC}$ and computational (open source) models, starting with pharmacokinetics/dynamics.

\section{Biology}

12. Define strategies to tackle issues on cell-to-cell variation, maturation, and stability of cells.

13. Standardize culture medium and explore the use of blood in single perfusion of multi-OoC.

14. Include immune and endocrine system components in OoC to improve physiological relevance.

15. Include gender-, origin- and age-related aspects in OoCs for representation of human diversity.

\section{Applications}

16. Define the type of throughput required for the application of OoCs.

17. Address personalized medicine (you-on-a-chip), clinical trials-on-chip and environmental toxicological assays as highest priority applications.

18. Involve the food industry to raise their interest in $\mathrm{OoC}$ applications.

19. Focus on toxicities or diseases for which no satisfactory pre-clinical models and treatment exist as a niche market for OoCs.

20. Combine a set of organs involved in ADME pathways that can match the critical functions needed for compound testing and studying transport of drugs and bioactive factors.

\section{Dissemination, communication and collaboration}

21. Keep expectations about the technology realistic when communicating with media and the public.

22. Develop a synergistic outreach based on careful communication of achievements and new developments by developers and stakeholders.

23. Involve patients, clinicians, companies and regulatory authorities early in the development of OoCs to ensure effective dialogue and avoid procedural obstacles to implementation.

24. Attract investors and funding agencies to support the development of prototypes into mass fabrication to meet the industrial demands of quality and reproducibility.

25. Build the network and realize integrative programs, collaborative projects, consortia, a digital platform, and international funding strategies for the development of $\mathrm{OoC}$ technology.

26. Foster next generation researchers: organize exchanges among institutes, trainings, workshops and conferences to nurture talents as prime accelerators for the progress of OoC technology.

\section{References}

Abaci, H. E. and Shuler, M. L. (2015). Human-on-a-chip design strategies and principles for physiologically based pharmacokinetics/pharmacodynamics modeling. Integr Biol 7, 383-391. doi:10.1039/c4ib00292j

Abaci, H., Guo, Z., Doucet, Y. et al. (2017). Next generation human skin constructs as advanced tools for drug development. 
Exp Biol Med 242, 1657-1668. doi:10.1177/1535370217712690

Alépée, N., Bahinski, A., Daneshian, M. et al. (2014). State-ofthe-art of $3 \mathrm{D}$ cultures (organs-on-a-chip) in safety testing and pathophysiology. ALTEX 31, 441-477. doi:10.14573/altex. 1406111

Avior, Y., Sagi, I. and Benvenisty, N. (2016). Pluripotent stem cells in disease modelling and drug discovery. Nat Rev Mol Cell Biol 17, 170-182. doi:10.1038/nrm.2015.27

Bahinski, A., Horland, R., Huh, D. et al. (2015). The promise and potential of "organs-on-chips" as preclinical models. Appl In Vitro Toxicol 1, 235-242. doi:10.1089/aivt.2015.29002.rtl

Barrile, R., Meer, A. D., Park, H. et al. (2018). Organ-on-chip recapitulates thrombosis induced by an anti-CD154 monoclonal antibody: Translational potential of advanced microengineered systems. Clin Pharmacol Ther 104, 1240-1248. doi:10.1002/ cpt. 1054

Bellin, M., Marchetto, M. C., Gage, F. H. and Mummery, C. L. (2012). Induced pluripotent stem cells: The new patient? Nat Rev Mol Cell Biol 13, 713-726. doi:10.1038/nrm3448

Benam, K. H., Novak, R., Nawroth, J. et al. (2016). Matched-comparative modeling of normal and diseased human airway responses using a microengineered breathing lung chip. Cell Syst 3 , 456-466. doi:10.1016/j.cels.2016.10.003

Bhatia, S. N. and Ingber, D. E. (2014). Microfluidic organs-onchip. Nat Biotechnol 32, 760-772. doi:10.1038/nbt.2989

Brown, J. A., Pensabene, V., Markov, D. A. et al. (2015). Recreating blood-brain barrier physiology and structure on chip: A novel neurovascular microfluidic bioreactor. Biomicrofluidics 9, 054124. doi:10.1063/1.4934713

Brown, J. A., Codreanu, S. G., Shi, M. et al. (2016). Metabolic consequences of inflammatory disruption of the blood-brain barrier in an organ-on-chip model of the human neurovascular unit. J Neuroinflammation 13, 306. doi:10.1186/s12974-0160760-y

Cyr, K. J., Avaldi, O. M. and Wikswo, J. P. (2017). Circadian hormone control in a human-on-a-chip: In vitro biology's ignored component? Exp Biol Med 242, 1714-1731. doi: $10.1177 / 1535370217732766$

Del Rio, D., Zimetti, F., Caffarra, P. et al. (2017). The gut microbial metabolite trimethylamine- $N$-oxide is present in human cerebrospinal fluid. Nutrients 9, 1053. doi:10.3390/nu9101053

Esch, E. W., Bahinski, A. and Huh, D. (2015). Organs-on-chips at the frontiers of drug discovery. Nat Rev Drug Discov 14, 248260. doi:10.1038/nrd4539

Ewart, L., Dehne, E.-M., Fabre, K. et al. (2018). Application of microphysiological systems to enhance safety drug assessment in drug discovery. Ann Rev Pharmacol Toxicol 58, 65-82. doi:10.1146/annurev-pharmtox-010617-052722

Fatehullah, A., Tan, S. H. and Barker, N. (2016). Organoids as an in vitro model of human development and disease. Nat Cell Biol 18, 246-254. doi:10.1038/ncb3312

Franzen, N., van Harten, W. H., Retèl, V. P. et al. (2019). Impact of organ-on-a-chip technology on pharmaceutical R\&D costs. Drug Discov Today 24, 1720-1724. doi:10.1016/j.drudis. 2019.06.003

Henry, O. Y. F., Villenave, R., Cronce, M. J. et al. (2017). Organs- on-chips with integrated electrodes for trans-epithelial electrical resistance (TEER) measurements of human epithelial barrier function. Lab Chip 17, 2264-2271. doi:10.1039/c71c00155j

Low, L. A. and Tagle, D. A. (2017). Organs-on-chips: Progress, challenges, and future directions. Exp Biol Med 242, 15731578. doi: $10.1177 / 1535370217700523$

Mak, I. W., Evaniew, N. and Ghert, M. (2014). Lost in translation: Animal models and clinical trials in cancer treatment. Am J Transl Res 6, 114-118.

Marx, U., Andersson, T. B., Bahinski, A. et al. (2016). Biologyinspired microphysiological system approaches to solve the prediction dilemma of substance testing. ALTEX 33, 272-321. doi:10.14573/altex.1603161

Maschmeyer, I., Lorenz, A. K., Schimek, K. et al. (2015). A fourorgan-chip for interconnected long-term co-culture of human intestine, liver, skin and kidney equivalents. Lab Chip 15, 2688-2699. doi:10.1039/c5lc00392j

Mastrangeli, M., Millet, S., Mummery, C. L. et al. (2019). Building blocks for a European organ-on-chip roadmap. ALTEX 36, 481-492 doi:10.14573/altex.1905221

McGonigle, P. and Ruggeri, B. (2014). Animal models of human disease: Challenges in enabling translation. Biochem Pharmacol 87, 162-171. doi:10.1016/j.bcp.2013.08.006

Mermoud, Y., Felder, M., Stucki, J. D. et al. (2018). Microimpedance tomography system to monitor cell activity and membrane movements in a breathing lung-on-chip. Sensor Actuat B: Chem 255, 3647-3653. doi:10.1016/j.snb.2017.09.192

Middelkamp, H. H. T., van der Meer, A., Marjan Hummel, J. et al. (2016). Organs-on-chips in drug development: The importance of involving stakeholders in early health technology assessment. Appl In Vitro Toxicol 2, 74-81. doi:10.1089/ aivt.2015.0029

Osaki, T., Uzel, S. G. M. and Kamm, R. H. (2018). Microphysiological 3D model of amyotrophic lateral sclerosis (ALS) from human iPS-derived muscle cells and optogenetic motor neurons. Sci Adv 4, eaat5847. doi:10.1126/sciadv.aat5847

Pampaloni, F., Reynaud, E. G. and Stelzer, E. H. K. (2007). The third dimension bridges the gap between cell culture and live tissue. Nat Rev Cell Biol 8, 839-845. doi:10.1038/nrm2236

Ronaldson-Bouchard, K. and Vunjak-Novakovic, G. (2018). Organs-on-a-chip: A fast track for engineered human tissues in drug development. Cell Stem Cell 22, 310-324. doi:10.1016/j. stem.2018.02.011

Roussel, B., Clerc, S. and Villien, M. (2018). Organs-on-chips report: From technologies to market. http://www.yole.fr/ OrgansOnChips_Market.aspx\#.XOOuNFIzaUk (accessed 26. 08.2019).

Smirnova, L., Kleinstreuer, N., Corvi, R. et al. (2018). 3S - Systematic, systemic, and systems biology and toxicology. ALTEX 35, 139-162. doi:10.14573/altex.1804051

Sutherland, J. J., Jolly, R. A., Goldstein, K. M. and Stevens, J. L. (2016). Assessing concordance of drug-induced transcriptional response in rodent liver and cultured hepatocytes. PLoS Comput Biol 12, e1004847. doi:10.1371/journal.pcbi.1004847

van de Stolpe, A. and Kauffmann, R. H. (2015). Innovative human-specific investigational approaches to autoimmune dis- 
ease. RSC Advances 5, 18451-18463. doi:10.1039/c4ra15794j

van Meer, B. J., de Vries, H., Firth, K. S. A. et al. (2017). Small molecule absorption by PDMS in the context of drug response bioassays. Biochem Biophys Res Commun 482, 323-328. doi:10.1016/j.bbrc.2016.11.062

Vernetti, L., Gough, A., Baetz, N. et al. (2017). Functional coupling of human microphysiology systems: Intestine, liver, kidney proximal tubule, blood-brain barrier and skeletal muscle. Sci Rep 7, 42296. doi:10.1038/srep44517

Watson, D. E., Hunziker, R. and Wikswo, J. P. (2017). Fitting tissue chips and microphysiological systems into the grand scheme of medicine, biology, pharmacology, and toxicology. Exp Biol Med 242, 1559-1572. doi:10.1177/1535370217732765

Wikswo, J. P., Block, F. E. $3^{\text {rd }}$, Cliffel, D. E. et al. (2013a). Engineering challenges for instrumenting and controlling integrated organ-on-chip systems. IEEE Trans Biomed Eng 60, 682-690. doi:10.1109/tbme.2013.2244891

Wikswo, J. P., Curtis, E. L., Eagleton, Z. E. et al. (2013b). Scaling and systems biology for integrating multiple organs-on-a-chip. Lab Chip 13, 3496-3511. doi:10.1039/c31c50243k

Wikswo, J. P. (2014). The relevance and potential roles of microphysiological systems in biology and medicine. Exp Biol Med 239, 10612-1072. doi:10.1177/1535370214542068

Xiao, S., Coppeta, J. R., Rogers, H. B. et al. (2017). A microfluidic culture model of the human reproductive tract and 28day menstrual cycle. Nat Commun 8, 14584. doi:10.1038/ ncomms 14584

Zhang, B. and Radisic, M. (2017). Organ-on-a-chip devices advance to market. Lab Chip 17, 2395-2420. doi:10.1039/ c61c01554a

Zhang, B., Korolj, A., Lai, B. F. L. and Radisic, M. (2018). Advances in organ-on-a-chip engineering. Nat Rev Mater 3, 257278. doi:10.1038/s41578-018-0034-7

Zhang, Y. S., Aleman, J., Shin, S. R. et al. (2017). Multisensor-in- tegrated organs-on-chips platform for automated and continual in situ monitoring of organoid behaviors. Proc Natl Acad Sci U $S$ A 114, e2293-e2302. doi:10.1073/pnas.1612906114

\section{Acknowledgements}

The authors would like to thank the 17 experts interviewed for the ORCHID WP2, and all other experts that contributed to ORCHID and/or took part in the ORCHID Vision Workshop (full list of ORCHID partners and affiliations in Section A.2 of Appendix ${ }^{2}$ ); Aurore Puech (CEA, France) for assistance in the bibliometric analysis; Berend van Meer (Leiden University Medical Center, The Netherlands) for notetaking during the ORCHID Vision Workshop; and Nora Franzen (University of Twente, The Netherlands) for previewing early results of the ORCHID WP3's impact study at the Vision Workshop. The authors also thank John Wikswo and Christine Mummery for their valuable contributions to the revision and improvement of the manuscript.

This work was funded by the European Union's Horizon 2020 Research and Innovation program under grant agreement No. 766884, and by the Netherlands Organ-on-Chip Initiative, an NWO Gravitation project funded by the Ministry of Education, Culture and Science of the government of the Netherlands (024.003.001).

\section{Massimo Mastrangeli ${ }^{1 \#}$, Sylvie Millet ${ }^{2 \#}$, the ORCHID} partners ${ }^{3}$ and Janny van den Eijnden-van Raaij ${ }^{4}$

${ }^{1}$ Electronic Components, Technology and Materials (ECTM), Department of Microelectronics, Delft University of Technology, Delft, The Netherlands; ${ }^{2}$ Direction de la Valorisation, Service Bibliométrique et Etude Marketing, Université Grenoble Alpes, CEA, Grenoble, France; ${ }^{3}$ full list in Appendix; ${ }^{4}$ Institute for Human Organ and Disease Model Technologies (hDMT), Eindhoven, The Netherlands

\footnotetext{
\# contributed equally
} 